\title{
Search for L5 Earth Trojans with DECam
}

\author{
Larissa Markwardt $^{\oplus},{ }^{1 \star}$ D. W. Gerdes,${ }^{1,2}$ R. Malhotra ${ }^{\oplus},{ }^{3}$ J. C. Becker ${ }^{\oplus}, 1,4$ \\ S. J. Hamilton ${ }^{2}$ and F. C. Adams ${ }^{1,2}$ \\ ${ }^{1}$ Department of Astronomy, University of Michigan, 1085 South University Avenue, Ann Arbor, MI 48109, USA \\ ${ }^{2}$ Department of Physics, University of Michigan, 450 Church Street, Ann Arbor, MI 48109, USA \\ ${ }^{3}$ Lunar and Planetary Laboratory, The University of Arizona, 1629 E University Boulevard, Tucson, AZ 85721, USA \\ ${ }^{4}$ Division of Geological and Planetary Sciences, California Institute of Technology, 1200 E California Blvd, Pasadena, CA 91125, USA
}

Accepted 2019 December 19. Received 2019 December 17; in original form 2019 April 30

\begin{abstract}
Most of the major planets in the Solar system support populations of co-orbiting bodies, known as Trojans, at their L4 and L5 Lagrange points. In contrast, Earth has only one known co-orbiting companion. This paper presents the results from a search for Earth Trojans (ETs) using the DECam instrument on the Blanco Telescope at CTIO. This search found no additional Trojans in spite of greater coverage compared to previous surveys of the L5 point. Therefore, the main result of this work is to place the most stringent constraints to date on the population of ETs. These constraints depend on assumptions regarding the underlying population properties, especially the slope of the magnitude distribution (which in turn depends on the size and albedo distributions of the objects). For standard assumptions, we calculate upper limits to a 90 per cent confidence limit on the L5 population of $N_{\mathrm{ET}}<1$ for magnitude $H<15.5, N_{\mathrm{ET}}=60-85$ for $H<19.7$, and $N_{\mathrm{ET}}=97$ for $H=20.4$. This latter magnitude limit corresponds to Trojans $\sim 300 \mathrm{~m}$ in size for albedo 0.15. At $H=19.7$, these upper limits are consistent with previous L4 ET constraints and significantly improve L5 constraints.
\end{abstract}

Key words: methods: observational - minor planets, asteroids: general.

\section{INTRODUCTION}

The population of Earth Trojans (ETs) represents an important (if perhaps not abundant) part of our Solar system because it can provide constraints on the Solar system's dynamical history. This paper reports the results from a new observational survey that searches for ETs near the L5 Lagrange point of the Earth-Sun system. The survey uses the Dark Energy Camera (DECam) instrument on the Blanco $4 \mathrm{~m}$ telescope. Although no new objects have been detected, this observational program provides the strongest constraints to date on the population of ETs.

\subsection{Significance of Earth Trojans}

Despite their relative proximity to Earth compared to other astronomical objects, the ET population is poorly understood. ETs are asteroids that orbit near the Earth-Sun L4 or L5 Lagrange points $\left(60^{\circ}\right.$ ahead or behind the Earth, respectively). Trojan asteroids have been discovered accompanying other planets, including Venus, Mars, Jupiter, Uranus, and Neptune (Ivezić et al. 2001; Morbidelli et al. 2005; Almeida, Peixinho \& Correia 2009; de la Fuente Marcos \& de la Fuente Marcos 2014; de la Fuente Marcos \& de la Fuente Marcos 2017; Yoshida \& Terai 2017; Lin et al. 2019). In contrast, only one ET has been found, $2010 \mathrm{TK}_{7}$, which was discovered serendipitously by WISE (Connors, Wiegert \& Veillet 2011).

Characterization of this population is important as ETs can give a unique insight into our Solar system. Numerical simulations have shown that ETs can have orbits near the L4 or L5 points that are stable on time-scales of order the current age of the Earth (Tabachnik \& Evans 2003; Malhotra \& Jeongahn 2011; Ćuk, Hamilton \& Holman 2012; Marzari \& Scholl 2013). As a result, the objects in this population could be undisturbed remnants from our primordial protoplanetary disc. In principle, the distribution of ETs can thus be used to constrain Solar system formation models. The chemical composition of these bodies could also provide insight into the properties of the primordial material from which our Solar system formed.

ETs have also been studied as potential lunar impactors. In particular, there appears to be an asymmetry of rayed lunar craters on the leading and trailing sides of the moon (Morota \& Furumoto 2003). Both Gallant, Gladman \& Ćuk (2009) and Ito \& Malhotra (2010) used numerical simulations to assess whether this asymmetry could be due to impacts of Near Earth Asteroids (NEAs). Gallant et al. (2009) concluded that, within the uncertainties of their simulations and of the observational data, the known population of NEAs were sufficient to account for the observed crater asymmetry, whereas 
Ito \& Malhotra (2010) found that the known NEA population could only account for $\sim 50$ percent of the asymmetry. An unobserved population of NEAs with very small velocities relative to the moon, like Earth co-orbitals, has been suggested and studied as a solution to this discrepancy (Ito \& Malhotra 2010; JeongAhn \& Malhotra 2010).

However, due to the lack of observational constraints on the ET population, the viability of ETs as this missing impactor population has been investigated only through theoretical models of the population, which may not represent the true distribution of co-orbitals. Detections of the ET populations in both the L4 and L5 Lagrange points - or robust upper limits - will provide important constraints on planet formation theories, which consider both the dynamical evolution and the physical properties of rocky Solar system objects.

\subsection{An unstable Earth Trojan: $2010 \mathrm{TK}_{7}$}

ETs are a particularly difficult population to observe; the L4 and L5 points are always at low solar elongations as observed from the Earth $\left(\sim 60^{\circ}\right.$ ahead or behind the Sun, respectively), meaning they can only be observed around dawn or dusk when the sky is still relatively bright. Moreover, due to the geometries of their orbits, ETs are never observed at opposition, and their larger phase angles make them even fainter and harder to detect.

These challenges may be part of the reason that only one ET, $2010 \mathrm{TK}_{7}$, has been observed to date (Connors et al. 2011). However, the orbit of this object is not consistent with primordial ETs, which are expected to be long-term stable librators near L4 or L5. In particular, $2010 \mathrm{TK}_{7}$ has a large amplitude tadpole orbit, librating between Earth and L3 (the Lagrange point behind the Sun), rather than remaining near L4 (Connors et al. 2011; Dvorak, Lhotka \& Zhou 2012). We consider such orbits distinct from primordial ETs, though it is possible for these orbits to also be stable on the order of $\sim \operatorname{Myr}$ (Dvorak et al. 2012; Marzari \& Scholl 2013).

Numerical integrations indicate that $2010 \mathrm{TK}_{7}$ has a highly chaotic and short-lived orbit. Estimated lifetimes vary from $\sim 7000$ yr (Connors et al. 2011) to about $\sim 0.25$ Myr (Dvorak et al. 2012). Taking into account the Yarkovsky effect, Zhou et al. (2019) also concluded that $2010 \mathrm{TK}_{7}$ is too small to maintain a long-term stable orbit. As a result, this object is most likely an asteroid that was temporarily captured as an Earth co-orbital, rather than a primordial ET.

\subsection{Previous ET searches}

There have been a few dedicated searches for ETs. The most recent searches were a ground-based survey for L5 ETs (Whiteley \& Tholen 1998, W\&T98), an OSIRIS-REx search as the spacecraft flew past the L4 point on its way to the asteroid Bennu (Cambioni et al. 2018, O-R18), and a search made by the Hayabusa2 spacecraft as it flew past the L5 point on its way to Ryugu (Yoshikawa et al. 2018). None of these surveys found any other ETs.

An upper limit calculation from the Hayabusa results has not yet been published (Yoshikawa et al. 2018). Whiteley \& Tholen (1998) placed an upper limit on the ET population of $\sim 3$ objects per square degree to $R=22.8$. Cambioni et al. (2018) placed an upper limit on the population of $73 \pm 22$ objects $\sim 210$ (S-type asteroids) to $\sim 470 \mathrm{~m}$ (C-type asteroids) in size. For comparison, they also applied their method to the Whiteley \& Tholen (1998) survey and found an upper limit of $194 \pm 116$ objects at the OSIRIS-REx limiting magnitude (Cambioni et al. 2018). These limits imply that there could still be tens to hundreds of undiscovered ETs. Moreover, the Whiteley \& Tholen (1998) search at L5 only covered a 0.35 sq deg area near L5. From these relatively large upper limits and limited survey coverage, it is clear that the ET population is far from being completely, or even sufficiently, characterized.

In this paper, we present the results of a new survey directed at the Sun-Earth L5 point with DECam on the Blanco $4 \mathrm{~m}$ telescope. In Section 2, we provide a description of the observations that make up our survey. In Section 3, we provide a description of the pipeline to reduce the data, extract moving objects, and create a catalogue of ET candidates. In Section 4, we present all of the moving objects found by our survey. In Section 5, we present the upper limits on the ET population derived from the results of our survey. In Section 6, we discuss our findings and their implications.

\section{DECAM SURVEY}

Our survey utilized the $V R$ filter on the DECam (Flaugher et al. 2015), which is a prime focus imager on the $4 \mathrm{~m}$ Blanco telescope at the Cerro Tololo Inter-American Observatory. The $V R$ filter is the widest filter on DECam, making it easier to detect faint ETs. The sky coverage for this survey is shown in Fig. 1. We observed shortly after sunset on the night of 2018 June 16, covering an area near Earth's L5.

DECam has 61 active CCD chips that cover 3 sq deg on the sky. As shown in Fig. 1, our survey consisted of eight fields, covering an area of 24 sqdeg ( $~ 70$ times larger than Whiteley \& Tholen 1998). See Table A1 in Appendix for complete list of exposures. Since stable ETs may have libration amplitudes of up to $30^{\circ}$ but low inclinations, the fields were arranged to proceed eastward from the L5 point within roughly $\pm 2^{\circ}$ of the ecliptic.

Two consecutive $15 \mathrm{~s}$ exposures were taken of each field, and this sequence was repeated 4 times at approximately $15 \mathrm{~min}$ intervals. The two short exposures were used to identify objects that were transient over $\sim 30$ s of readout time between exposures. Since ETs are expected to have a rate of motion across the sky of $\sim 1 \mathrm{deg} \mathrm{d}^{-1}$ (see Section 3.2.5), an exposure time of $15 \mathrm{~s}$ was chosen to avoid trailing of these objects in the individual images, which would make these objects harder to detect in the individual exposures. The subsequent visits were used to track the motion of the object and identify a tracklet for a potential Solar system object.

This sampling strategy did not allow us to fit an orbit to detected Solar system objects, because the observational arcs are too short. Therefore, we were not able to dynamically classify any of the objects found in our survey (as was done in Ivezić et al. 2001). Any new objects found in this survey would require additional follow-up to be classified as ETs, but they could be identified as ET candidates due to their characteristic rate of motion across the sky of $\sim 1 \mathrm{deg} \mathrm{\textrm {d } ^ { - 1 }}$ (see Section 3.2.5).

\section{REDUCTION PIPELINE}

We reduced the survey data and extracted Solar system objects, in particular ETs, by the following steps: ${ }^{1}$

(i) DECam Community Pipeline (Valdes et al. 2014): for basic data reduction.

\footnotetext{
${ }^{1}$ Code for this paper available at: https://github.com/markwardtla/EarthTroj
} anSearch-2018. 


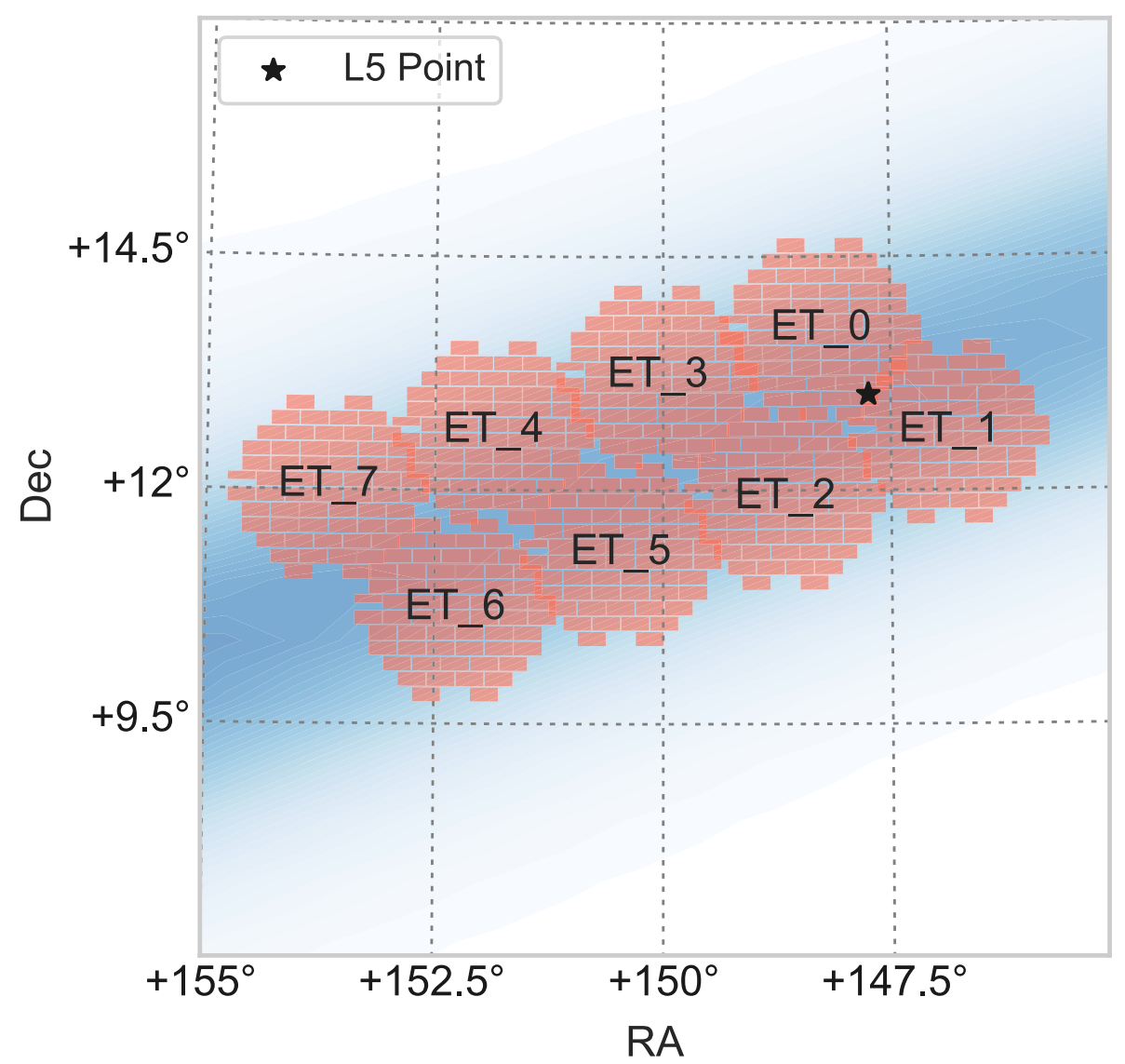

Figure 1. The area covered by our survey. The star marks the location of the L5 point. The relative density of synthetic ET objects (see Section 5.1) is shown in the background in blue. Each rectangle corresponds to a chip in one of the eight DECam fields. In total, these fields cover $\sim 24$ sq deg near L5.

(ii) SOURCE EXTRACTOR (Bertin \& Arnouts 1996): for source identification.

(iii) BULLSEYE: a pipeline we developed for this project to link transient sources.

(iv) Verification by eye/catalogue creation: for removal of false positives.

\subsection{SOURCE EXTRACTOR}

We used SOURCE EXTRACTOR (SE) to identify sources in each of the exposures. Table 1 shows the SE parameters used for this analysis. The DETECT_THRESH parameter was set to $1 \sigma$; it was set this low for completeness at very faint objects, as many anomalous sources could be identified/filtered out (see Sections 3.2.2 and 3.2.4).

In particular, after running SE, we remove any sources with a centre pixel flagged as bad by the DECam Community Pipeline. This pipeline flags bad/compromised pixels (e.g. known detector defects), saturated pixels, bleed trails, and transient artefacts (Valdes et al. 2014). We do not use the transient flags in order to ensure that we are not losing transient Solar system objects. This step removes hundreds of blatantly bad/compromised sources from each chip.

\subsection{BULLSEYE}

Once the SE catalogues for each exposure were created, we linked these sources together to identify transient Solar system objects, particularly ETs. Based on the cadence of our survey, the best possible detection of a Solar system object would be eight individual
Table 1. SOURCE EXTRACTOR parameters. The filter called 'all-ground' is a $3 \times 3$ convolution mask with $\mathrm{FWHM}=2$ pixels.

\begin{tabular}{ll}
\hline \multicolumn{2}{c}{ Extraction parameters } \\
DETECT_TYPE & CCD \\
DETECT_MINAREA & 6 \\
DETECT_THRESH & 1.0 \\
ANALYSIS_THRESH & 1.0 \\
FILTER & $Y$ \\
FILTER_NAME & All-ground \\
DEBLEND_NTHRESH & 32 \\
DEBLEND_MINCONT & 0.005 \\
CLEAN Weighting parameters & \\
\multicolumn{1}{c}{ Background parameters } \\
WEIGHT_TYPE & MAP_VAR \\
BACK_TYPE & AUTO \\
BACK_VALUE & 0.0 \\
BACK_SIZE & 256 \\
BACK_FILTERSIZE & 3 \\
\hline
\end{tabular}

detections (one for each exposure) in a nearly straight line, due to the short arc of these observations $(\sim 1 \mathrm{~h})$. We created BULLSEYE to identify sets of transient detections that fit to a line and have a speed consistent with nearby Solar system objects (see Table 2), and to flag those whose speed is consistent with ETs. This process 
Table 2. BULLSEYE parameters. $R$ is the stationary source radius (measured by SE). See Section 3.2 for discussion of these parameters.

Transient identification

$\begin{array}{ll}\text { Transient metric } & >0.25 \operatorname{arcsec} \\ \text { Stationary metric } & <0.5 \operatorname{arcsec} \\ \text { Min. detections for stationary } & 3 \\ \text { Bright stationary metric } & <2 \mathrm{arcsec} \\ \text { Min. detections for bright stationary } & 7 \\ \text { Transient ring min. radius } & R \\ \text { Transient ring max. radius } & 3.5 R \\ \text { Transient ring min. density } & 0.035 \text { sources per } \operatorname{arcsec}^{2}\end{array}$

Linking

$\begin{array}{ll}\text { Min. detections for link } & 5 \\ \text { Min. speed } & 0.1 \mathrm{deg} \mathrm{d}^{-1} \\ \text { Max. speed } & 2.0 \mathrm{deg} \mathrm{d}^{-1} \\ \text { Cluster eps } & 0.05 \\ \text { Min samples for cluster } & 4\end{array}$

Flagging ET candidates

Min. speed

Max. speed

$0.75 \operatorname{deg~} \mathrm{d}^{-1}$ $1.25 \mathrm{deg} \mathrm{d}^{-1}$

is run for each survey field, chip by chip. We do not attempt to link objects that crossed chip boundaries; from our recovery rate of known Solar system objects, we determined that chip-crossing objects are a small fraction of the population and some may still have linkable observations in at least one of the chips. The steps in this pipeline are described in more detail in the following sections.

\subsubsection{Identify transient sources}

The pipeline starts by identifying sources from the SE catalogues that are transient between exposures. Using ASTROPY's match_to_catalog (Astropy Collaboration 2013, 2018), we compare the catalogue of the sources between each of the sets of two exposures. If the distance to the nearest source between the two catalogues is more than the transient metric (i.e. there is no source at the same position in the next exposure, see Table 2), the source is considered to be transient. This comparison must actually be done both ways (i.e. compare the first catalogue to the second and vice versa) because it is possible for a transient source to only show up in the second of the two catalogues due to differences in observing conditions between exposures. Only objects that are transient between the sets of two exposures will continue on to the next step.

\subsubsection{Cut stationary sources}

Stationary objects (sources with the same position in a set of two exposures) should not be included in the transient catalogue after the previous step. However, stationary sources with inconsistent astrometry or faint stationary sources which were not detected in one of the two exposures in a set could have been erroneously included in the transient catalogue and needed to be removed. We use ASTROPY's search_around_sky method (Astropy Collaboration 2013 , 2018) to compare objects in the transient catalogue from the previous step to an SE catalogue, which includes the source detections in all of the exposures for a field. If a transient object's position matches to too many objects in the full SE catalogue within the search radius (see Table 2), it will be cut. In other words, this step removes objects which have the same position in three out of the eight exposures. To avoid cutting true transients, we choose parameters for these cuts that are more lenient to avoid removing actual transients from our search. We also removed rings of scattered light around stationary sources that meet the 'bright stationary' criteria in Table 2.

\subsubsection{Connect transients}

After cuts to the transient catalogue, we link these detections between exposures together to identify potential Solar system objects. Detections are linked if their motion between exposures is consistent with the motion of a Solar system object. In particular, we assumed a min and max speed typical of nearby Solar system objects, primarily main belt asteroids (see Table 2), but did not assume a direction of motion. There are classes of Solar system objects that would not make our speed cut, such as very nearby asteroids or distant, outer Solar system objects. However, neither of these populations are the focus of this search, and the expected ET speed of $1 \mathrm{deg} \mathrm{d}^{-1}$ is well within our search space.

With a min and max speed but no restriction on direction, the search space for another detection is an annulus. Therefore, for each transient detection (the 'anchor' transient), we used search_around_sky to identify other transient objects that fall within that search annulus in each of the subsequent exposures. Anchor transients can come from any of the exposures to account for sources near our limiting magnitude that were not detected in the first exposure and objects near the edge of the chip. At the end of this step, we have a list of transient sources that are linked to subsequent detections that would be consistent with the rate of motion of Solar system objects.

\subsubsection{Fit lines of motion}

If all of the detections connected to an anchor transient from the previous step were of the same Solar system object, we would expect them to have the same velocity at each observation. Here, we define the velocity as the object's rate of motion across the sky in $\mathrm{deg}^{-1}$ (speed) and position angle (direction) as measured with respect to the anchor point. Therefore, all of these transient detections need to have similar velocities in order to identify them as the same object.

To group these detections by velocity, we used DBSCAN ('Density-based spatial clustering of applications with noise') from the SCIKIT-LEARN package (Pedregosa et al. 2011). This method is able to identify regions with a high density of points in Cartesian space; specifically, a cluster must have at least some minimum number of points within a certain distance (see 'eps' parameter in Table 2). However, the velocities of our transient detections are in polar coordinates (a speed with an angular direction). Therefore, we converted the speed and position angle into a Cartesian space to then identify detections with clustered velocities. If this cluster contained at least five detections from different exposures of the linked object, we add it to our transient object catalogue. Therefore, in the end we have a final set of detections that are linked together as a candidate tracklet.

\subsubsection{Flag ETs}

Due to the short arcs of our investigations, it was not possible to fit orbits to our detections, which would be required to definitively classify an object as an ET. However, due to their relatively consistent position with respect to the Earth and Sun, simulated 

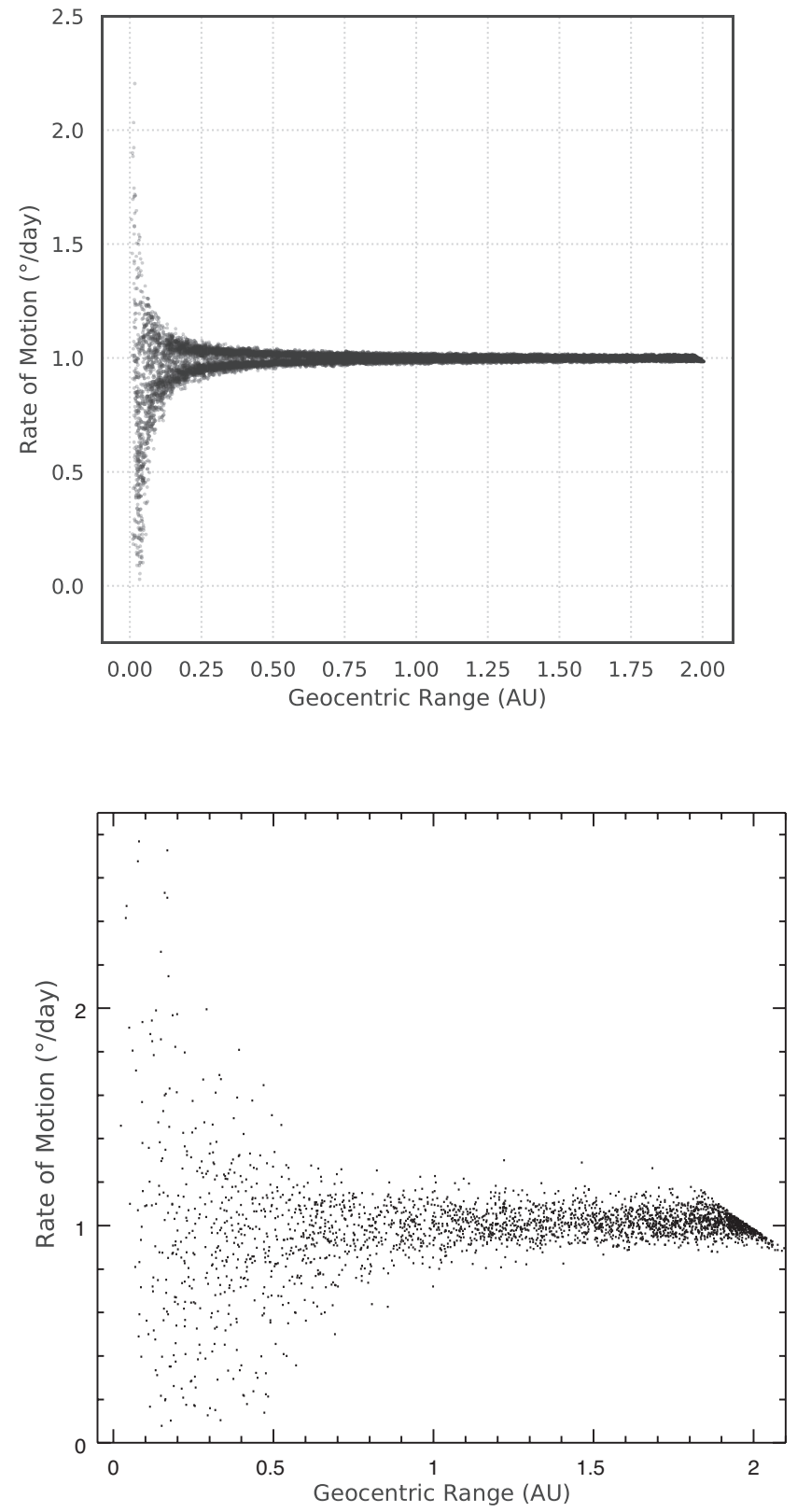

Figure 2. Top: The rate of motion on the sky for the 'Strict' set of synthetic ETs (see Section 5.1). Other than at small geocentric ranges $(\lesssim 0.1 \mathrm{au})$, the ETs have a well constrained speed of $1 \mathrm{deg}^{-1}$. Bottom: Same as Top but for the 'Jupiter Trojan' set of synthetic ETs (synthetic ETs generated based on observations of Jupiter Trojans; see Section 5.1). These objects also have a well constrained speed of $\sim 1 \mathrm{deg} \mathrm{d}^{-1}$ for geocentric ranges $>0.5 \mathrm{au}$. There is more dispersion in the speed, but this is expected as this set of ETs was generated based off of observations of Jupiter Trojans.

ETs (see Section 5.1) have a distinct rate of motion on the sky of $1 \mathrm{deg} \mathrm{d}^{-1}$, except at small geocentric ranges (see Fig. 2). Therefore, any previously undiscovered object in our catalogue that has a mean speed between 0.75 and $1.25 \mathrm{deg} \mathrm{d}^{-1}$ is considered an ET candidate in our survey.

However, the only known ET, $2010 \mathrm{TK}_{7}$, often has rates of motion that are not consistent with this assumption (see Fig. 3). While technically its mean rate of motion is nearly $1 \mathrm{deg} \mathrm{d}^{-1}$, it is clear that this object most often moves at $\sim 0.75 \mathrm{deg} \mathrm{d}^{-1}$. Fortunately, this slower rate of motion often still falls within our speed assumption.
Moreover, there are many evenings when $2010 \mathrm{TK}_{7}$ would not have actually been detectable due to high airmass, solar elongation, moon phase, etc. For a large portion of the evenings where $2010 \mathrm{TK}_{7}$ would have been detectable by our survey (if it had been directed towards L4), $2010 \mathrm{TK}_{7}$ would still have been flagged as an ET by our pipeline (if it had been detected). Regardless, $2010 \mathrm{TK}_{7}$ does not have an orbit that is representative of primordial ETs, and it should not be considered as a particularly strong or valid test for this pipeline. Thus, we consider our assumption of ET candidates having a speed between 0.75 and $1.25 \mathrm{deg} \mathrm{d}^{-1}$ to be reasonable since it is consistent with simulated ET populations and roughly valid for $2010 \mathrm{TK}_{7}$ (a notably atypical ET).

However, an object that has a $1 \mathrm{deg} \mathrm{d}^{-1}$ rate of motion is not guaranteed to be an ET. There are other populations of asteroids that can have similar rates of motion and could happen to overlap with the L5 point (see Section 4.3). Again, additional observations of a candidate would be required to actually constrain its orbit and classify it as an ET.

\subsection{Verification by eye/catalogue creation}

Despite the cuts on bad detections, transients, and links, false positives were still found by the BULLSEYE code. Therefore, linked objects were reviewed by hand in order to remove obvious false positives from our catalogue. Such objects included detections of stationary objects with very variable astrometry between exposures, detections in the scattered light rings around very bright objects, detections along diffraction spikes, and detections along detector defects. Out of the 7676 candidate objects identified by the pipeline, 6059 were determined to be false positives. However, many of these anomalous objects were linked to large rings of scattered light around very bright or detector defects making them easy to identify and remove from our catalogue.

While reviewing the candidate objects, it was also convenient to look for objects that were identified by SE and correspond to previously known Solar system objects but were not linked by the pipeline (i.e. non-recovered objects). Many of these objects were observed to be in crowded field, confused with a bright star, or fell in a chip gap.

\section{DETECTED OBJECTS}

\subsection{Previously known objects}

To determine which objects had been previously discovered, we compared our catalogue to the SkyBoT data base (Berthier et al. 2006). In total, there were 4126 minor bodies coincident with our survey area, and we recovered detections of 1546 known Solar system objects ( $\sim 40$ per cent). The majority of these objects were main belt asteroids. All of these detections have been submitted to the Minor Planet Center (MPC). ${ }^{2}$

Fig. 4 shows our recovery of known objects as a function of their $V$-band magnitude as predicted by SkyBoT. As expected, our recovery rates drop off at fainter magnitudes. In particular, the recovery rates are $\gtrsim 50$ per cent for $V \lesssim 22$, and they drop quickly to 0 percent beyond this point. This implies that overall limiting magnitude for our survey is roughly $V=22$. However, reducing this limit to a single number obfuscates the different detection thresholds in each image due to differences in chips, weather conditions, etc.

\footnotetext{
${ }^{2}$ minorplanetcenter.net
} 


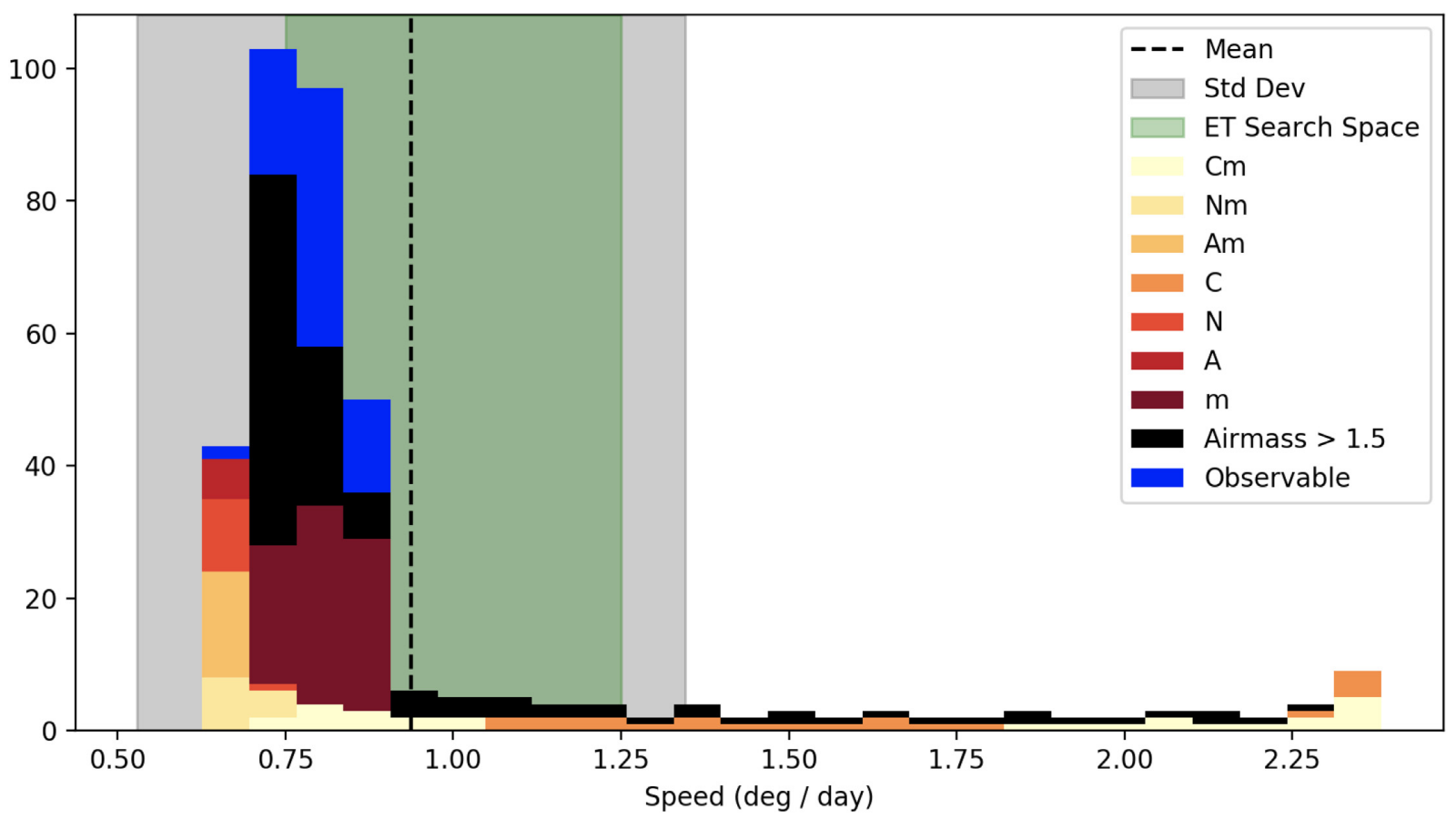

Figure 3. This figure shows the predicted rates of motion for $2010 \mathrm{TK}_{7}$ on each night in 2018 (taken from JPL HORIZONS: ssd.jpl.nasa.gov/?horizons). The dashed line shows the mean speed and the grey region depicts the standard deviation. The green region represents the speeds for which an object would be flagged as an ET by our pipeline. Nights on which we consider $2010 \mathrm{TK}_{7}$ to be observable are plotted in blue. The other colours represent nights on which observing $2010 \mathrm{TK}_{7}$ would be unfavourable according to the Solar Presence and Lunar Presence parameters from JPL HORIZONS. In particular, 'C' is civil twilight/dawn, ' $\mathrm{N}$ ' is nautical twilight/dawn, ' $\mathrm{A}$ ' is astronomical twilight/dawn, and ' $\mathrm{m}$ ' is refracted upper limb of Moon on or above apparent horizon. Although the standard deviation region extends beyond our assumptions for ET speed, the mean is well with our search space. In fact, most of the nights beyond our speed cuts are ones for which $2010 \mathrm{TK}_{7}$ is not observable.

This limiting magnitude also explains why the overall recovery rate for our survey was only 40 per cent as a majority of the previously known objects were not detectable in our survey.

Fig. 4 also shows our recovery rates as a function of their rate of motion as predicted by SkyBoT. The recovery rates in each speed bin are roughly consistent with the overall recovery rate. Therefore, we conclude we are not especially more/less sensitive to detecting objects moving at a particular speed. However, these recovery rates are not entirely independent of speed; slow moving rates are somewhat lower than for fast moving objects. This discrepancy is likely due to the fact that slower moving objects are more likely to be flagged as stationary. Since ETs move at a speed of $\sim 1 \mathrm{deg} \mathrm{d}^{-1}$, they were very unlikely to be flagged as stationary in our data set. As is shown in Fig. 4, we have a higher recovery rate at larger speeds, meaning we may even be more efficient at recovering ETs.

Moreover, due to the nature of our survey, it is likely that we observed these objects at much higher phase angles than their discovery. Therefore, we expect our recovery rate and limiting absolute magnitude of known asteroids to be lower than an identical survey of objects at lower phase angles.

\subsection{New objects}

Table 3 lists all of the Solar system objects identified by our pipeline that did not match to positions of known asteroids (a total of 27 new objects). Most importantly, none of these objects have a rate of motion of $\sim 1 \mathrm{deg}^{-1}$. Rather, they are consistent with the rates we observed for main belt asteroids. Therefore, by our definition, we did not find any ET candidates in our survey. However, due to the short arcs of our data set, we cannot determine the orbits of these new objects or their classification. These detections have been submitted to the MPC so that they may be linked to an orbit in the future.

\subsection{Aten and Apollo asteroids}

While our pipeline did not flag any new objects as ET candidates, there were a few previously known objects that were flagged because they happened to have a rate of motion of $\sim 1 \mathrm{deg} \mathrm{d}^{-1}$ (see Table 4). One of these objects was an Apollo asteroid while the other was an Aten. We draw attention to these objects specifically because they provided a serendipitous test of our pipeline while also highlighting the limitations of our method.

First, Apollo and Aten asteroids both have Earth crossing orbits, with $q>1$ au and $q<1$ au, respectively. If these asteroids were to cross the Earth's orbit at a point near the L4 or L5 points with a rate of motion of $\sim 1 \mathrm{deg} \mathrm{d}^{-1}$ they would be indistinguishable from ET candidates in our survey. Fig. 5 shows the orbit of the flagged Aten asteroid, which indeed crosses the Earth's orbit near the L5 point and has a rate of motion of $1.17 \mathrm{deg}^{-1}$. The orbit for the flagged Apollo asteroid is similar. The fact that these distinct populations would be indistinguishable in our observations highlights the fact that our method cannot definitively classify transient objects as ETs.

However, it is still significant that these objects were flagged. Since these asteroids behave exactly as we expect ETs would in our data set and they were properly detected and flagged, this implies that our pipeline was capable of finding ETs as well. Certainly, these objects are not a robust test of the capability of the pipeline, but they did allow for a test on real, known objects. The only known ET that 

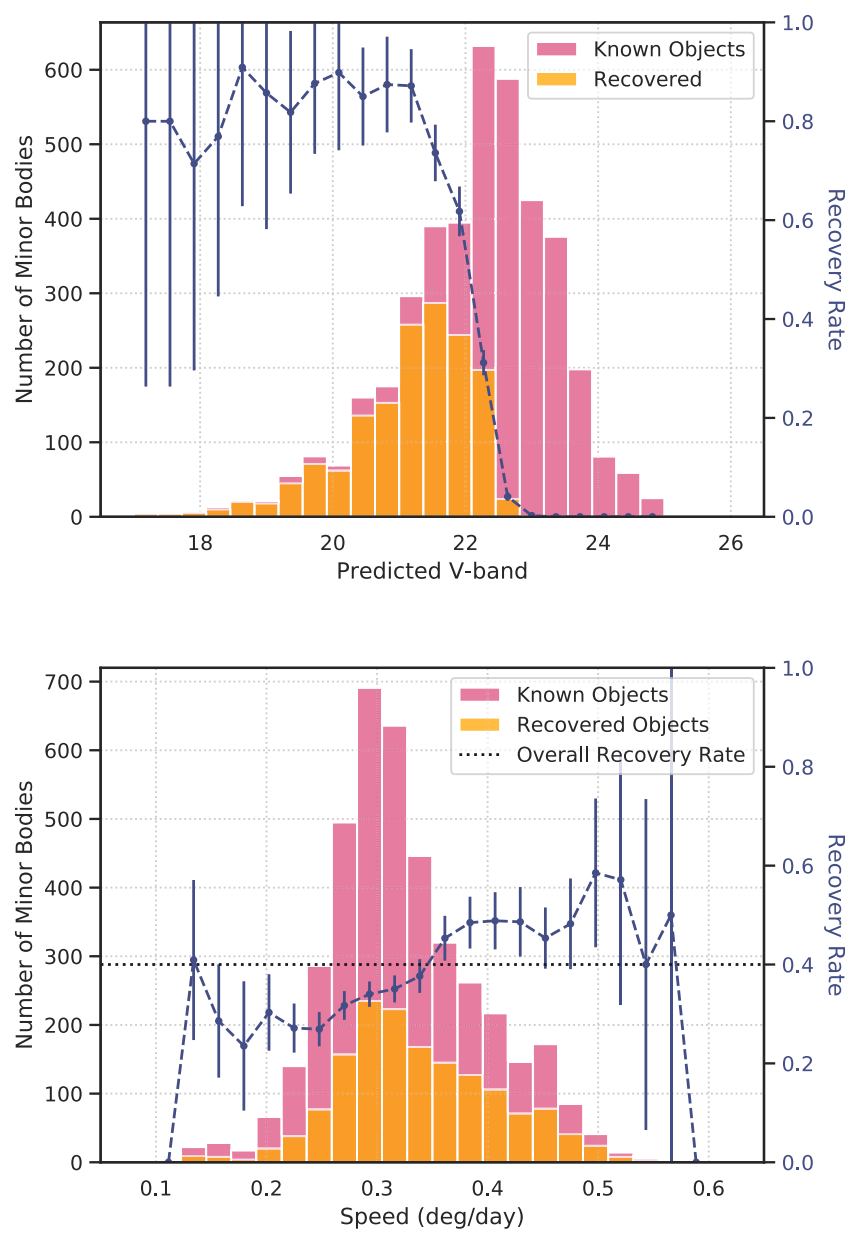

Figure 4. Top: Recovery rates for previously known minor bodies as a function of predicted $V$-band magnitude. The known minor bodies that coincided with our survey area are shown in pink, and those which were detected and linked by our pipeline are shown in yellow. The recovery rate in each bin, with Poisson errors, are shown as a blue dotted line. Bottom: This plot shows our recovery rates for previously known minor bodies as a function of predicted total sky motion. Colours are the same as Top. The overall recovery rate of $\sim 40$ percent is shown as a black dotted line. The recovery rates across all of these speeds are generally consistent with this overall rate, but recovery rates are lower for slower speeds.

we could have otherwise tested on $\left(2010 \mathrm{TK}_{7}\right)$ is at the $\mathrm{L} 4$ point, which is not within our survey area.

\section{UPPER LIMITS ON ET POPULATION}

To calculate an upper limit on the ET population, we generated a population of simulated ETs, injected them into our data set, ran these images through the pipeline as described in Section 3, and then measured our recovery rate for these objects. We then used this recovery rate to place an upper limit on the ET population.

\subsection{Synthetic ETs}

To generate synthetic ET populations, we adopt the restricted threebody model of Sun, Earth, and a test particle. We generated two different synthetic ET sets: a 'Strict' ET set and a 'Jupiter Trojan' ET set. Both sets were used to predict the rate of motion for ETs
Table 3. New objects. The individual observations for all of these objects have been submitted to the Minor Planet Center. Columns: (1) Our designation for this object. The first number is the field and the second number is the chip the object was found in. The third number is a unique id; (2) Inferred $V$-band magnitude for the object. See Section 5.1 for a description of how the $V$-band magnitudes were calculated; (3) Rate of sky motion measured for the object $\left(\operatorname{deg~d}^{-1}\right)$. The rate of motion is averaged over all of the detections; (4) Position angle measured for the object (deg). The P. A. is averaged over all of the detections; (5) Number of detections of the object.

\begin{tabular}{lccrc}
\hline Designation & $\mathrm{m}_{V}$ & Rate of motion & P. A. & Num. \\
\hline 1_55_5 & 23.1 & 0.29 & 129.3 & 6 \\
2_37_0 & 22.5 & 0.37 & 115.3 & 6 \\
2_51_2 & 22.6 & 0.40 & 99.9 & 7 \\
2_54_9 & 22.8 & 0.37 & 113.3 & 5 \\
3_21_2 & 23.0 & 0.49 & 110.9 & 6 \\
3_34_3 & 23.5 & 1.76 & 264.3 & 5 \\
4_15_13 & 22.6 & 0.15 & 122.3 & 7 \\
4_36_0 & 22.8 & 0.32 & 113.1 & 8 \\
4_39_6 & 23.4 & 0.27 & 99.7 & 5 \\
4_45_0 & 22.9 & 0.49 & 107.9 & 7 \\
4_56_5 & 23.3 & 0.14 & 129.1 & 5 \\
5_16_2 & 22.8 & 0.46 & 107.5 & 7 \\
5_32_1 & 23.2 & 0.32 & 102.5 & 6 \\
6_24_5 & 23.3 & 0.36 & 115.7 & 6 \\
6_34_0 & 22.9 & 0.32 & 121.5 & 8 \\
6_58_2 & 22.4 & 0.36 & 153.8 & 7 \\
7_3_6 & 23.3 & 0.42 & 107.9 & 5 \\
7_4_3 & 23.1 & 0.30 & 89.1 & 6 \\
7_15_1 & 23.0 & 0.32 & 130.9 & 6 \\
7_18_3 & 22.8 & 0.16 & 90.2 & 8 \\
7_19_2 & 22.9 & 0.36 & 105.3 & 7 \\
7_19_3 & 22.8 & 0.49 & 106.3 & 6 \\
7_20_3 & 23.2 & 0.38 & 113.9 & 6 \\
7_27_2 & 22.6 & 0.33 & 109.5 & 8 \\
7_33_5 & 23.2 & 0.39 & 114.3 & 5 \\
7_37_0 & 22.1 & 0.26 & 104.0 & 8 \\
7_50_1 & 22.6 & 0.33 & 129.3 & 7 \\
\hline
\end{tabular}

(see Section 3.2.5), but only objects from the Strict set were injected into our images.

\subsubsection{Strict ET set}

We generated a sample of $N=10^{6}$ Earth-like test particle orbits, with heliocentric osculating orbital elements as follows: semimajor axes uniform random in the range 0.99-1.01 au, eccentricities uniform random in 0-0.1, ecliptic inclinations uniform random in $0-0.1$ radians; the angular elements (longitude of ascending node, argument of perihelion, and true anomaly) uniform random values in the range $0-2 \pi$. We computed the position and velocity vectors, $\boldsymbol{r}$ and $\boldsymbol{v}$, in the barycentric frame, and the Jacobi integral for these particles

$C=2\left(\frac{1-\mu)}{\left|\boldsymbol{r}-\boldsymbol{r}_{\odot}\right|}+\frac{\mu}{\left|\boldsymbol{r}-\boldsymbol{r}_{\oplus}\right|}\right)+2 \hat{\boldsymbol{n}} \cdot(\boldsymbol{r} \times \boldsymbol{v})-v^{2}$,

where $\mu$ is Earth's mass as a fraction of the total mass (Earth and Sun) and $\hat{\boldsymbol{n}}$ is the unit vector normal to the ecliptic. We then selected the subset of those particles having Jacobi integral in the range $3-\mu$ to $3+\mu$, in standard units for the restricted three-body problem; this range of Jacobi integral covers the L4 and L5 libration zone. The synthetic L4 and L5 ETs were then selected as the subset having instantaneous positions within $30 \mathrm{deg}$ of heliocentric ecliptic 
Table 4. Previously known objects flagged as ETs. The individual observations for all of these objects have been submitted to the Minor Planet Center. Columns: (1) Object designation; (2) Orbit type taken from SkyBoT (Berthier et al. 2006); (3) Predicted $V$-band magnitude (taken from SkyBoT); (4) Rate of sky motion measured for the object $\left(\mathrm{deg} \mathrm{d}^{-1}\right)$. The rate of motion is averaged over all of the detections; (5) Position angle measured for the object (deg). The P. A. is averaged over all of the detections; (6) Number of detections of the object.

\begin{tabular}{lccccc}
\hline Designation & Orbit type & $\mathrm{m}_{V}$ & Rate of motion & P. A. & Num. detections \\
\hline 2003 HF2 & NEA:Apollo & 21.9 & 1.08 & 110.5 & 6 \\
2006 JF42 & NEA:Aten & 21.0 & 1.17 & 124.1 & 7 \\
\hline
\end{tabular}

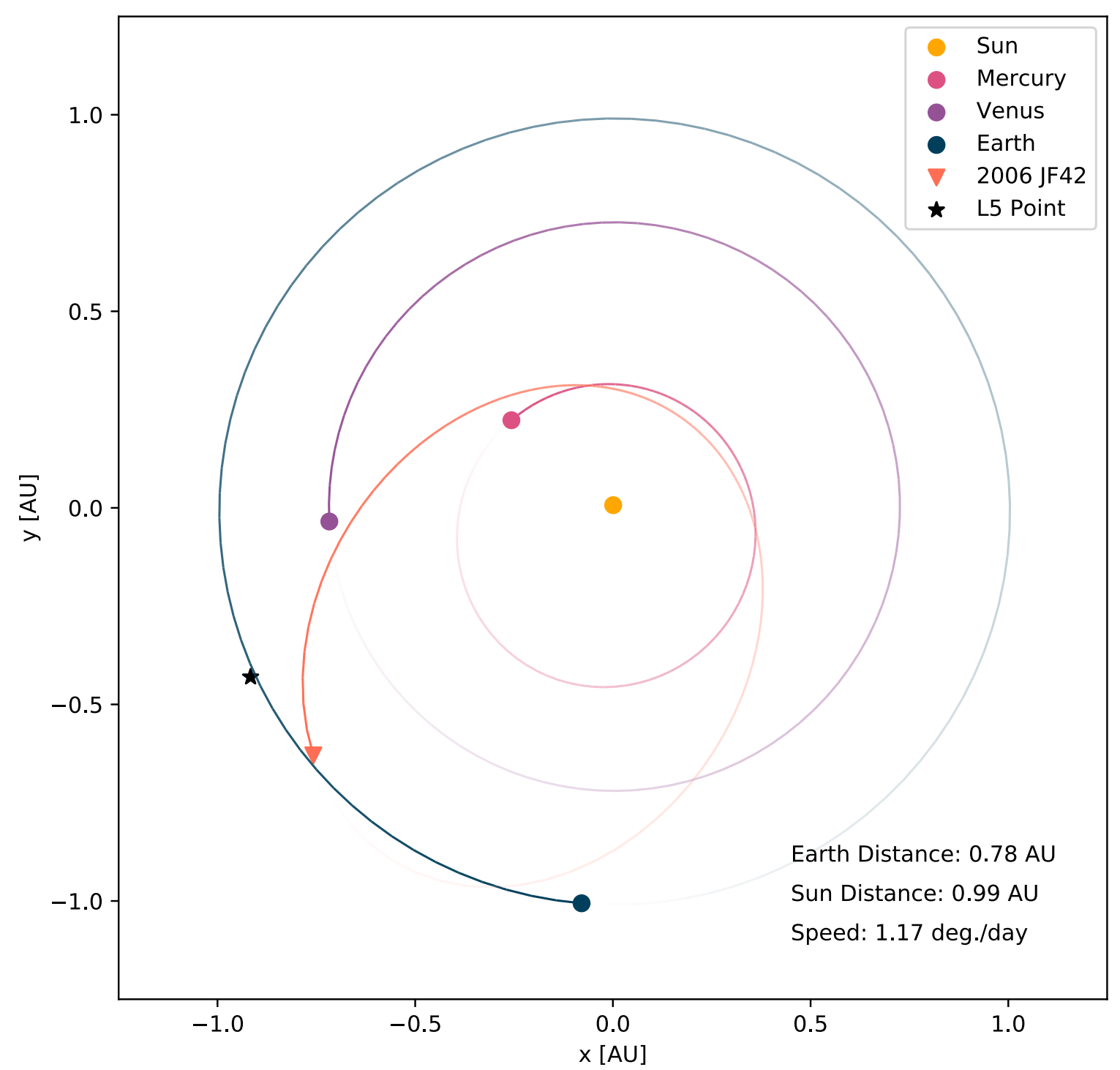

Figure 5. This figure shows the orbit of the Aten asteroid detected in our survey, $2006 \mathrm{JF}_{42}$. At the time of our observations, this asteroid was near the $\mathrm{L} 5$ point. With a distance to the Earth of 0.78 au and a distance to the Sun of 0.99 au, this object had a rate of motion of 1.17 deg d $\mathrm{d}^{-1}$, causing it to be misidentified as an ET candidate by our pipeline. This figure was produced using REBOUND (Rein \& Liu 2011).

longitude of the L4 point and L5 point, respectively. This produced a sample of 3797 L4 and 3726 L5 synthetic ETs.

\subsubsection{Jupiter Trojan ET set}

We started with the full data set of minor planet orbital data from the MPC (on 2018 November 27). We selected the observationally complete set of Jupiter Trojans from this set, which we defined as those objects of absolute magnitude $H<13.5$ and semimajor axis in the range 5.0-5.4 au (2844 object in total). To generate ET orbits, we made three changes to the orbital data set of Jupiter Trojans. First, we rescaled the semimajor axes of the particles to Earth's

$a_{\mathrm{ET}}=a_{\oplus}+\left(a-a_{\mathrm{Jupiter}}\right)\left(R_{\mathrm{H}, \oplus} / R_{\mathrm{H}, \text { Jupiter }}\right)$, 
where $R_{\mathrm{H}, \oplus}, R_{\mathrm{H}, J u p i t e r}$ are the Hill radii of Earth and Jupiter, respectively. Second, we computed the orbital plane orientation of each Jupiter Trojan relative to Jupiter's osculating orbital plane and reassigned it to be relative to Earth's osculating orbital plane. Third, we assigned the mean anomaly of ETs as follows:

$M_{\mathrm{ET}}=M+\lambda_{\oplus}-\lambda_{\text {Jupiter }}$,

where $\lambda_{\oplus}, \lambda_{\text {Jupiter }}$ are the mean longitudes of Earth and Jupiter at the epoch of the MPC's data, and $M$ is the mean anomaly of a Jupiter Trojan. These changes produce a population of synthetic ET orbits that has the same dispersion in semimajor axis, eccentricity, and inclination as the Jupiter Trojans, but relative to Earth's orbit and scaled to the size of Earth's Trojan regions.

\subsection{Injection of synthetic ETs}

To estimate our recovery rate for ETs, we drew from the Strict synthetic ET set and injected corresponding sources into our images (see footnote 1). To most accurately simulate the ET population in our images, the synthetic sources must be at realistic positions, follow a reasonable absolute magnitude $(H)$ and physical size distribution, and have the correct amount of injected counts for the corresponding magnitude.

The synthetic sources were injected as Gaussian point sources into the image array count by count. Note, assuming an albedo of 0.15 , we expect ETs to be less than about $1 \mathrm{~km}$ in size; such objects would not be resolved in our data set, so we can safely model them as point sources. Each synthetic source is generated to have the same full width at half-maximum (FWHM) as derived by the DECam Community pipeline for each image. In particular, the width $\sigma$ of a Gaussian distribution can be derived from the FWHM via the relation

$\mathrm{FWHM}=2 \sqrt{2 \ln 2} \sigma$.

Each count was added at a random $x$, $y$ shift from the centre of the object generated from this distribution. This method worked well for many sources, but not all; very bright sources often had a much larger angular size than the inferred FWHM (see Fig. 6).

To calculate the sky positions of our synthetic ETs, we used the generated heliocentric osculating orbital elements (see Section 5.1) and PYEPHEM ${ }^{3}$ to calculate the predicted RA, Dec. at the time of each of our observations. Our observing location at Cerro Tololo InterAmerican Observatory (longitude: $-70.80^{\circ}$, latitude: $-30.17^{\circ}$, elevation: $2207 \mathrm{~m}$ ) was taken into account for these calculations.

For the $H$ distribution, we use one similar to that of NEOs/asteroids

$N(<H)=A * 10^{\alpha H}$,

where $H$ is the absolute magnitude, $A$ is a normalization factor, and $\alpha$ is a slope parameter. We draw uniformly from a range of typical $\alpha$ $=0.46-0.54$ (Jedicke \& Metcalfe 1998; Bottke et al. 2000; Harris \& D'Abramo 2015; JeongAhn \& Malhotra 2015). We set $A$ such that $N\left(<H_{\max }\right)$ is equal to the total number of synthetic ETs. Of course, this means that we need to pick an $H_{\max }$; we defined our distribution to have $H_{\min }=15$ and $H_{\max }=20.5$. Our choice of $H_{\min }$ is somewhat arbitrary as very bright objects will be very rare in this distribution. Based on our recovery of previously known Solar system objects (see Section 4.1), we estimated our limiting magnitude to be $\sim 21.5$ in $V$ band, but we still recovered known objects at $V \sim 22.5$ (see

\footnotetext{
${ }^{3}$ rhodesmill.org/pyephem/
}
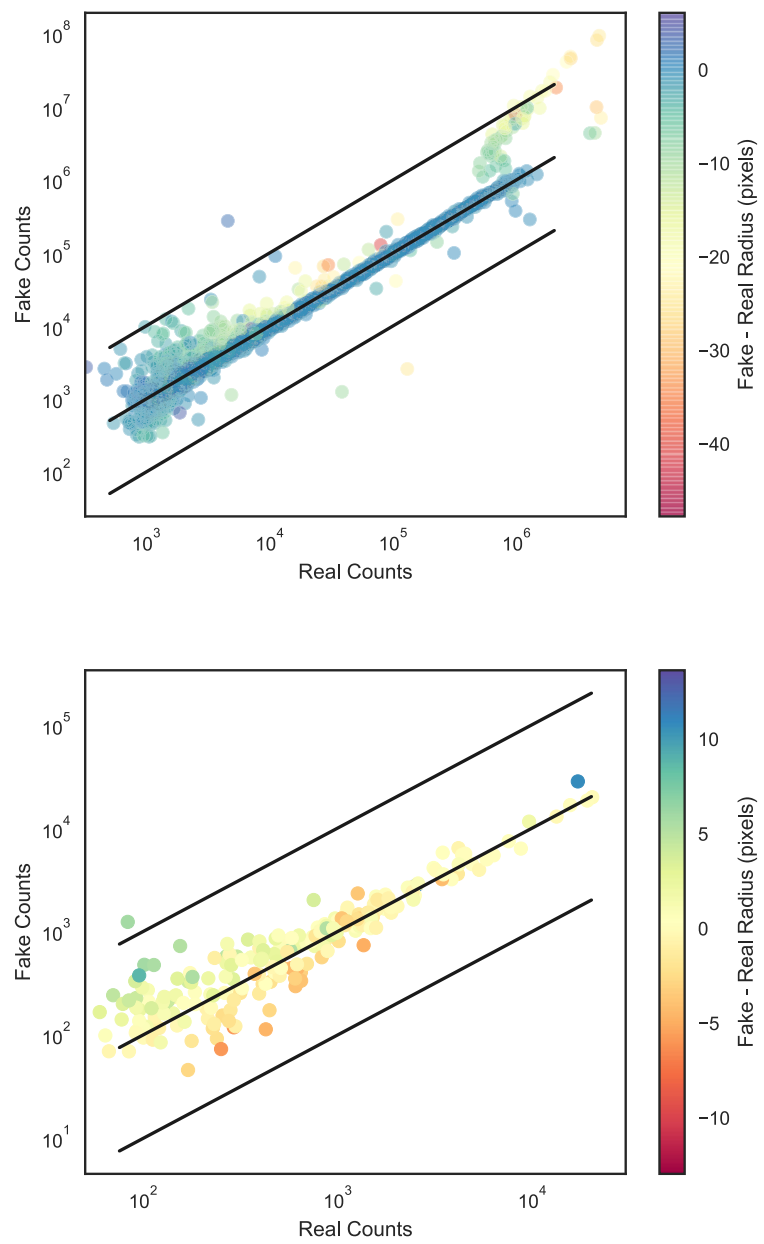

Figure 6. Top: This figure compares the number of counts measured by SE for Solar-type stars in Field 0 to the number of counts for a fake source of the same $V$-band magnitude. In general, the points lie well along the one-to-one line and the radii of the fake and real sources are nearly the same. Not surprisingly, there is more dispersion from this line at the faint end, but the points still have the same number of counts within an order of magnitude. There is also a clear overabundance of fake counts for the brightest objects, which is likely due to the saturation limit. Bottom: Same as Top but for known Solar system objects in Field 0. These points fall well along the one-to-one line and the radii of the real sources as compared to the fake ones are still nearly the same. Based on this plot, we conclude that our fake sources are consistent with our observations of known Solar system objects in our survey.

Section 4.1). This magnitude corresponds to $H \sim 20.4$. Therefore, we inject synthetic ETs up to $H_{\max }=20.5$.

To generate a magnitude for each specific object, we cannot use equation (5) directly as it is a cumulative distribution. Instead, we normalize equation (5) by $A$, such that $N\left(<H_{\max }\right)=1$ and then use this distribution as a probability distribution. Fig. 7 demonstrates that this method leads to magnitudes that are consistent with the theoretical distribution (i.e. the cumulative distribution of our randomly drawn magnitudes matches the theoretical distribution well). Therefore, we conclude that this method is sufficient to generate synthetic ETs with an accurate distribution.

Since each object's apparent magnitude is highly dependent on its location in its orbit, we use the functionality in PYEPHEM to calculate an apparent magnitude for the synthetic ETs at the time of each observation. To convert these magnitudes into counts, we 


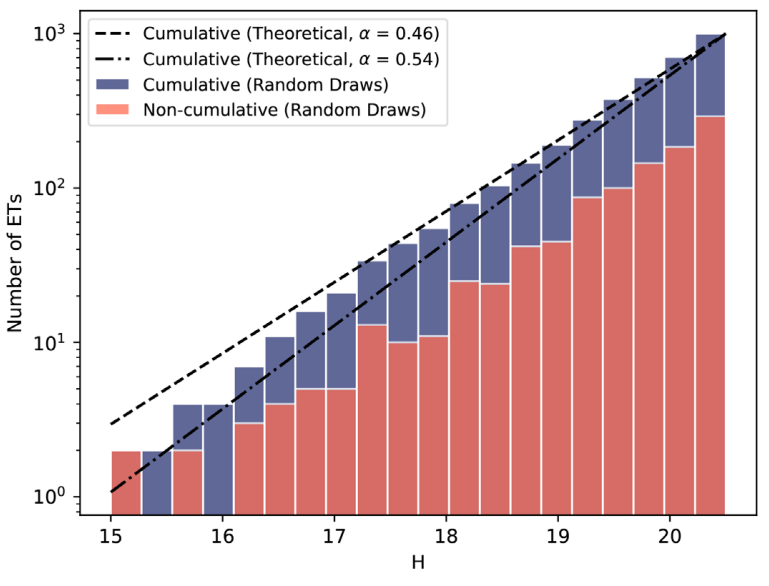

Figure 7. This figure compares our theoretical $H$ distribution of ETs (equation 5) to the distribution generated by random draws from equation (5) normalized by the total number of fake ETs (in the case of this figure, $10^{3}$ ). The black dashed and dot-dashed lines represent the theoretical $H$ distribution with $\alpha=0.46$ and 0.54 , respectively. The blue and red histograms represent the cumulative and non-cumulative $H$ distributions, respectively, which were generated for the simulated ETs (see Section 5.2 for description). Due to the random nature of this method, these distributions are similar to those used for the sources that were actually injected into our images to assess our recovery rate, but they are not exactly the same. Most importantly, the simulated cumulative distribution lies well between the two theoretical distributions. We therefore conclude that the injected ETs have an $H$ (and thus size) distribution consistent with what we would expect theoretically.

assume a linear relationship between counts and the flux of the source. This function will be slightly different between each chip as they are not all identical. Therefore, chip by chip, we identify SDSS sources in each image using ASTROQUERY (Ginsburg et al. 2019) and then fit a line to their counts as measured by SE (see Section 3.1) versus the apparent $V$-band magnitude. We calculated the $V$-band magnitude from the SDSS $g$ and $r$ magnitudes using

$V=g-0.59 *(g-r)-0.01$

(Jester et al. 2005). We used $V$-band magnitudes in order to compare to the predicted $V$-band magnitudes from SkyBoT (Berthier et al. 2006) for the known Solar system objects in our image.

One may expect that our fitted lines should have slopes of -0.4 . However, we found that restricting our fit to have a slope of this value led to synthetic sources with counts that were systematically higher than the real sources. Since the light observed from asteroids in optical wavelengths is primarily reflected sunlight, we compared the fitted line to only SDSS sources with solar-like colours: $g-r=$ $+0.44 \pm 0.02$. We found that these fits consistently overpredicted the number of counts for faint sources. We suspect that this discrepancy is due to a breakdown in the assumption that $V R$ and $V$ magnitudes should be comparable. In particular, the $V R$ filter is essentially a very broad $r$ filter $(\lambda \sim 475-750 \mathrm{~nm})$ that overlaps with the $g$ band. Since solar-type objects are more red $(g-r=$ +0.44 ), this implies that the flux overlapping with the $g$ band would be lower than expected assuming the object had a flat spectrum over $V R$. Therefore, the overlap with the $g$ band would be overpredicted, leading to consistently higher flux counts overall for solar-type objects. Therefore, we instead fit a line to only the solar-type SDSS objects in our images without imposing a slope constraint.

Fig. 6 shows the final comparison between the real counts for SDSS sources and Solar system object sources versus the measured counts for their synthetic counterparts. In both cases, most of the sources follow the one-to-one line, and nearly all of the synthetic sources have a number of counts within an order of magnitude of the real counts. There is more deviation at the faint end, but this is expected since this regime is more dominated by noise. There is a noticeable systematic surplus of counts for synthetic SDSS objects at the bright end (Fig. 6). This discrepancy is likely due to the saturation limit; while real sources cannot have a number of counts exceeding this limit, there is no such restriction for the synthetic objects, allowing them to have increasingly more counts as compared to the real sources. Since we do not see this problem at the faint end or for the Solar system objects, we do not attempt to correct for it. Also, in most cases, the angular size of the synthetic sources is nearly the same as the real source. Since the synthetic objects have similar sizes and counts compared to the real sources, we conclude that our method is able to inject realistic synthetic objects into our data set.

\subsection{Upper limit on ET Population}

To calculate an upper limit on the ET population, we injected the Strict set of synthetic ET objects (see Section 5.1) following the methods described in Section 5.2. Of course, only sources that fell within the survey area could actually be injected into our images; 886 of the 20555 objects were injected into our images (see Fig. 8). This means that our survey covered 4.3 per cent of this population. However, our survey only covered the L5 point, meaning we have no direct constraints on the L4 population. Therefore, we calculated our upper limits for the L5 population only. Since there were 3726 L5 ETs in the full synthetic set, our survey coverage of L5 ETs specifically is 24.0 percent. However, assuming that the L4 and L5 points are symmetric, our upper limit is applicable to the L4 population as well.

We then ran these images with synthetic ETs through our pipeline (Section 3) and counted the number of injected ETs that were then detected, linked, and flagged as an ET candidate. Fig. 9 shows the recovery rate as a function of $H$. These plots are representative of the efficiency of our pipeline and our detection limit. Of note, there were seven injected ET that were linked by the pipeline but were not flagged as ETs due to having speeds inconsistent with our $1 \mathrm{deg} \mathrm{d}^{-1}$ assumption. These objects are a small fraction of the nearly 1000 synthetic ETs injected into our images and were expected for synthetic objects with a small geocentric range (see Fig. 2). These objects are not included in our recovery rate, as we would not have considered them to be an ET candidate.

Overall, we recovered about 50 percent of the injected ETs. As expected, our recovery rate is much higher for the brighter objects and becomes very low at the faint end (12.8 percent at $H=20.4$ ). In Fig. 9, our limiting absolute magnitude appears to be $H \sim 20$; however, $H$ does not correspond directly to the $V$-band magnitudes of the injected ETs.

From these recovery rates, we can calculate an upper limit on the ET population adopting a Frequentist approach. Since we did not detect any ET candidates in our survey, by Poisson statistics, detecting three objects is within $3 \sigma$ of our result. Therefore, we calculate our limit as

$U(H)=\frac{3}{R(H) * C}$,

where $U(H)$ is our calculated upper limit, $R(H)$ is the recovery rate as a function of $H$ (see Fig. 9) and $C$ is the coverage of the L5 cloud for our survey (see Fig. 8). Again, $R(H)$ accounts for the 


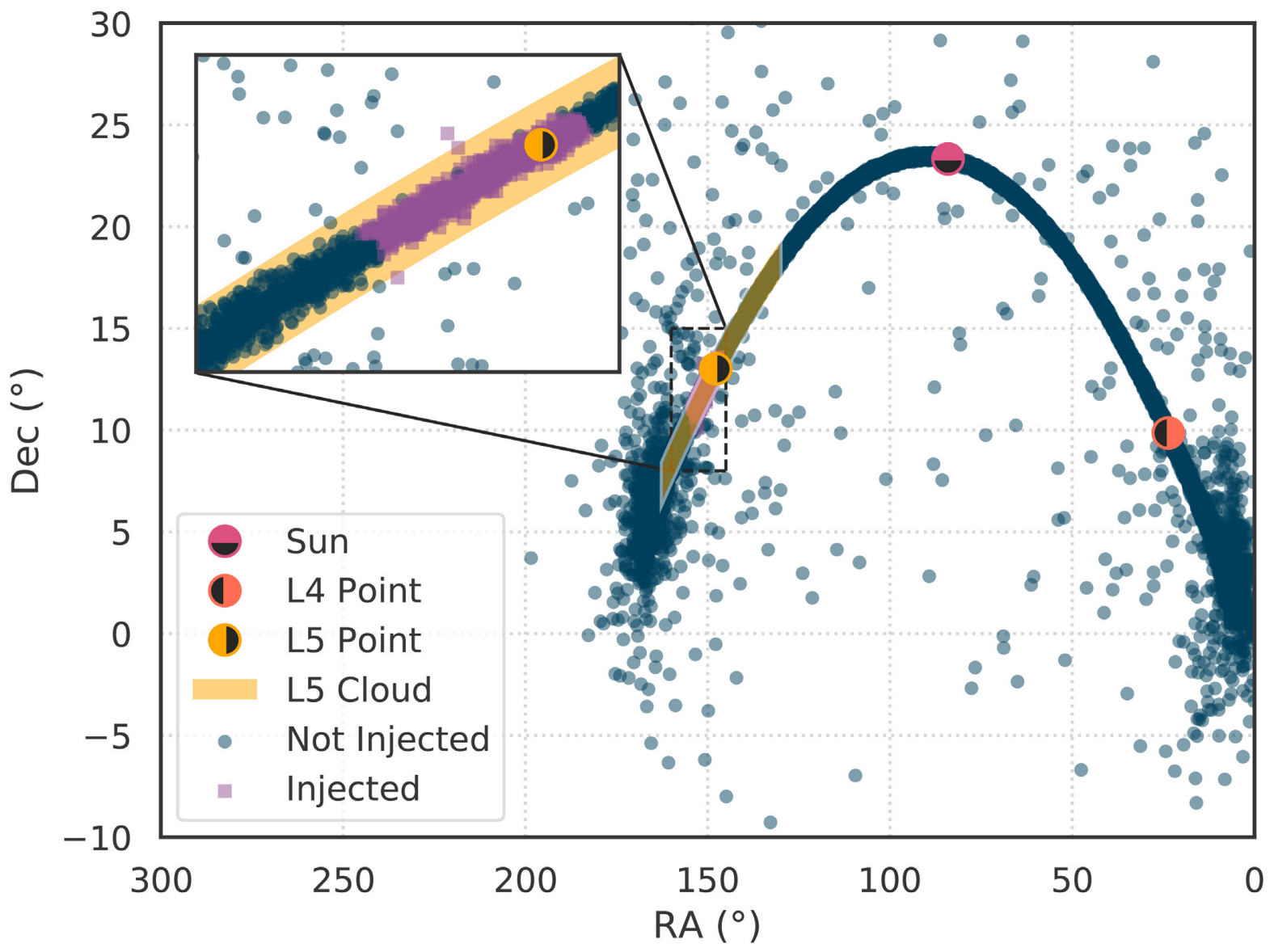

Figure 8. This figure demonstrates coverage by our survey of the synthetic ET population. The Lagrange points and the Sun are plotted as half filled circles for reference. The yellow shaded area represents the L5 cloud (the area with ecliptic longitude within 30 deg of L5). Synthetic ETs (see Section 5.1) that were injected into our images are plotted as purple squares, while those that were not are blue circles. These injected points essentially depict the area covered by our survey. These objects make up 24 per cent of the ETs in the L5 cloud.

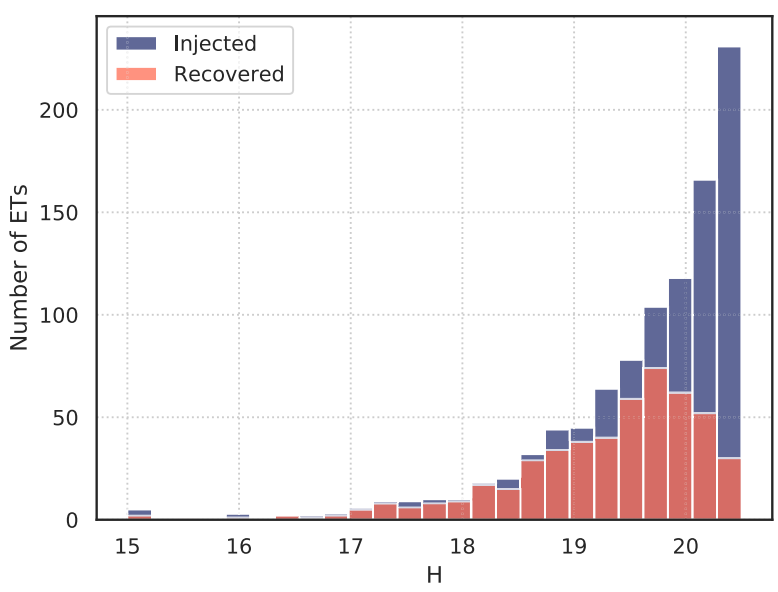

Figure 9. This figure compares the injected ETs (blue) from the set of Synthetic ETs to those that were detected, linked, and flagged as ET candidates by our pipeline (red); in other words, this plot shows our recovery rates as a function of $H$. We have nearly 100 per cent recovery rates for most of the bins at the bright end, and the rates begin to fall off at $\sim H=19.7$. This value is essentially our limiting magnitude in $H$ (though this does not directly relate to our limiting magnitude in $V$ band). Overall, we recovered $\sim 50$ per cent of the injected ETs. performance of our pipeline and our limiting magnitude while $C$ accounts for the limited area of our survey.

Fig. 10 shows our calculated upper limits as a function of $H$ and of size (assuming an albedo of 0.15 ). We also plot previously measured/estimated upper limits on the ET population at $H=20.5$. There are two distinct regimes in this recovery rate: flat at the bright end and sharp growth at the faint end. The upper limit becomes significantly higher at the faint end due to our limiting magnitude. In other words, the recovery rate becomes so low for the faintest objects that we only have relatively poor constraints on the population. Regardless, our calculated upper limit for the faintest $H$ bin, $H$ $=20.4(\sim 300 \mathrm{~m})$, is 97 . The bright end is essentially flat because the recovery rate was nearly 100 percent for these bins; since $C$ is the same for all bins and the recovery rate nearly the same, the calculated upper limit is constant $(\sim 15)$.

If we assume that the underlying $H$ distribution of the ET population follows a power law of index similar to that of the reported distributions for NEOs and main belt asteroids, we can calculate even more stringent (but model-dependent) upper limits for magnitudes in the flat regime. However, the true ET population may not follow such a distribution. If the ET population is primarily comprised of temporarily captured asteroids or NEOs, this assumption is likely reasonable. However, if the ET population is more similar to the ancient asteroid belt, it could be much shallower 


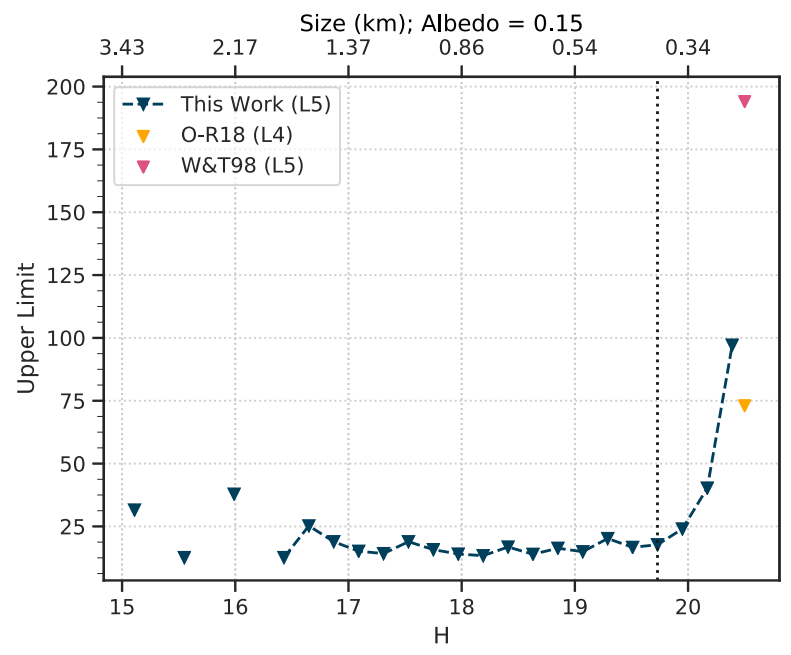

Figure 10. Upper limits on the ET population calculated from our survey area coverage (Fig. 8) and recovery rate (Fig. 9). The yellow triangle depicts the upper limit calculated for L4 ETs by Cambioni et al. (2018), while the pink triangle is the upper limit extrapolated from the Whiteley \& Tholen (1998) results by Cambioni et al. (2018). The upper limits from this work have two distinct regimes: flat at the bright end and steeply increasing at the faint end. We get a sharp increase for the faintest magnitudes due to our limiting magnitude and relatively poor recovery rates for faint ETs. For the faintest bin, $H=20.4$, we calculate an upper limit of $97 \mathrm{ETs}$. This limit is clearly more stringent than that from the Whiteley \& Tholen (1998) results (194) and somewhat higher than the Cambioni et al. (2018) limit (73) for L4. Our recovery rates are flat at the bright end because all of these bins had nearly 100 per cent recovery rates leading to a constant upper limit $(\sim 15)$ for those magnitudes. The dotted line represents the faintest magnitude $(H=19.7)$ still within the flat regime. At this magnitude, we calculate an upper limit of 18

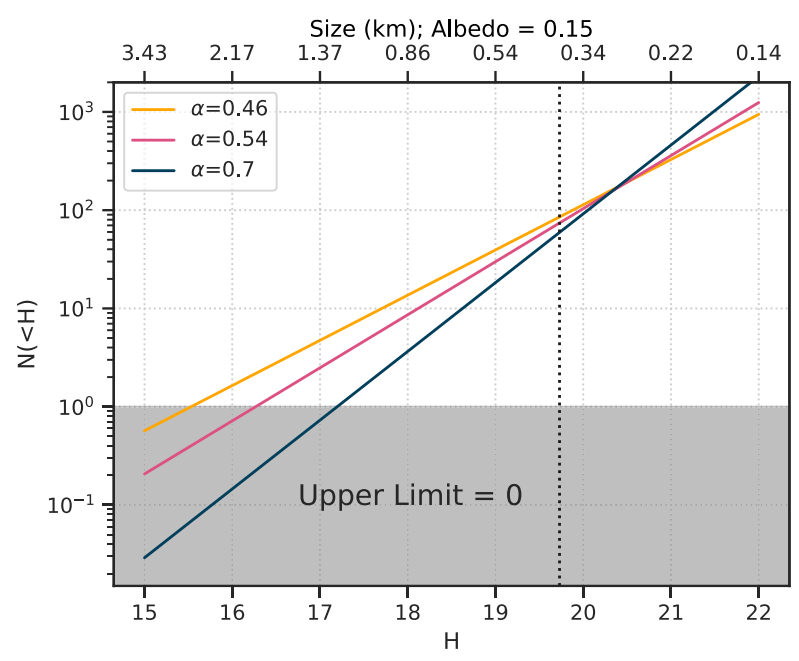

Figure 11. This figure depicts the stricter upper limits calculated for bright ETs assuming the ET $H$ distribution follows a power law (equation 5). Slopes of $0.46-0.54$ are consistent with the $H$ distributions for main belt asteroids. A slope of 0.7 was used in Cambioni et al. (2018) for similar extrapolations to compare their results to Whiteley \& Tholen (1998). The dotted line represents the point used for extrapolation, $H=19.7$ (see Fig. 10). The grey region depicts where $N(<H)<1$, meaning the upper limit is essentially 0 ETs. Note, $\alpha=0.46$ gives the most conservative upper limits of the three power laws at the bright end, while $\alpha=0.7$ is most conservative at the faint end.
(Tsirvoulis et al. 2018) or depend on its collisional history (Bottke et al. 2005). There is also evidence for differences in albedo and size distributions amongst NEO subgroups themselves (Mainzer et al. 2012). With our current sparse observational constraints, it is not possible to take these complexities into account; we instead assume the population follows a single power law of index in the range $0.46-0.7$.

Following this assumption, we calculate a cumulative $H$ distribution as a power law normalized to our calculated upper limit for the faintest $H$ bin still in the flat regime (corresponding to the dotted line in Fig. 10). We assume $\alpha=0.46$ and 0.54 as before (see Section 5.2) and 0.7 for comparison to Cambioni et al. (2018; see Section 6.2). Fig. 11 upper limits calculated by extrapolation equation (5) assuming each of these power laws. It is clear that with this method, we obtain much more stringent constraints on bright ETs than in Fig. 10; we even obtain an upper limit of $<1$ ET at $H$ $\lesssim 15.5,16.3,17.2$ for $\alpha=0.46,0.54,0.7$, respectively. We also calculate an upper limit of $\sim 60-85$ (depending on the power law assumed) ETs with $H<19.7(d \sim 390 \mathrm{~m}$ assuming an albedo of $0.15)$. Note that $\alpha=0.46$ gives the most conservative upper limits for bright/large objects, whereas $\alpha=0.7$ gives the most conservative limits at the faint end.

\section{DISCUSSION}

\subsection{Summary}

In this work, we present the results of our search for L5 ETs using the $V R$ filter on DECam. In total, our survey covered 24 sq deg near the Earth-Sun L5 point. Our survey only consisted of $\sim 1 \mathrm{~h}$ of observations, so the arcs for any observed Solar system objects were not long enough to fit an orbit or dynamically classify these objects. However, ETs are expected to have a distinctive rate of motion on the sky of $1 \mathrm{deg} \mathrm{d}^{-1}$ (Fig. 2). Therefore, we developed the BULLSEYE code to link together transient objects in our exposures and flag any tracklets with a similar speed as an ET candidate.

With this survey, we found 27 tracklets that do not correspond to any previously known Solar system objects (see Table 3). However, none of these objects were flagged as ET candidates. Based on this non-detection, we place upper limits (see Figs 10 and 11) on the ET population of $\sim 60-85$ ETs with $H<19.7$ (corresponding to a size of $\sim 390 \mathrm{~m}$ assuming an albedo of 0.15 ) and 97 ETs with $H=20.4$ $(\sim 290 \mathrm{~m})$.

\subsection{Comparison to previous surveys}

There have been three previous dedicated searches for ETs (Whiteley \& Tholen 1998; Cambioni et al. 2018; Yoshikawa et al. 2018). No ETs were found and no upper limits have been published in the Hayabusa2 search, so we can only compare to W\&T98 and O-R18. W\&T98 was similar to our search in that it was ground-based and covered L5, but it covered a much smaller area $(\sim 0.35 \mathrm{sq} \mathrm{deg})$. The O-R18 search was quite different in that it was conducted as the probe flew through the Lagrange point and it covered L4 rather than L5. However, we are still able to make comparisons to their results as we would expect the populations at the two Lagrange points to be symmetric.

We first compared our faintest upper limit (at $H=20.4$ ) to the upper limits for W\&T98 and O-R18 as calculated in Cambioni et al. (2018; see Fig. 10). Our upper limit of 97 ETs is slightly higher than the O-R18 result of 73 ETs at L4, but clearly more stringent than the upper limit calculated based on the W\&T98 search (194 ETs). 


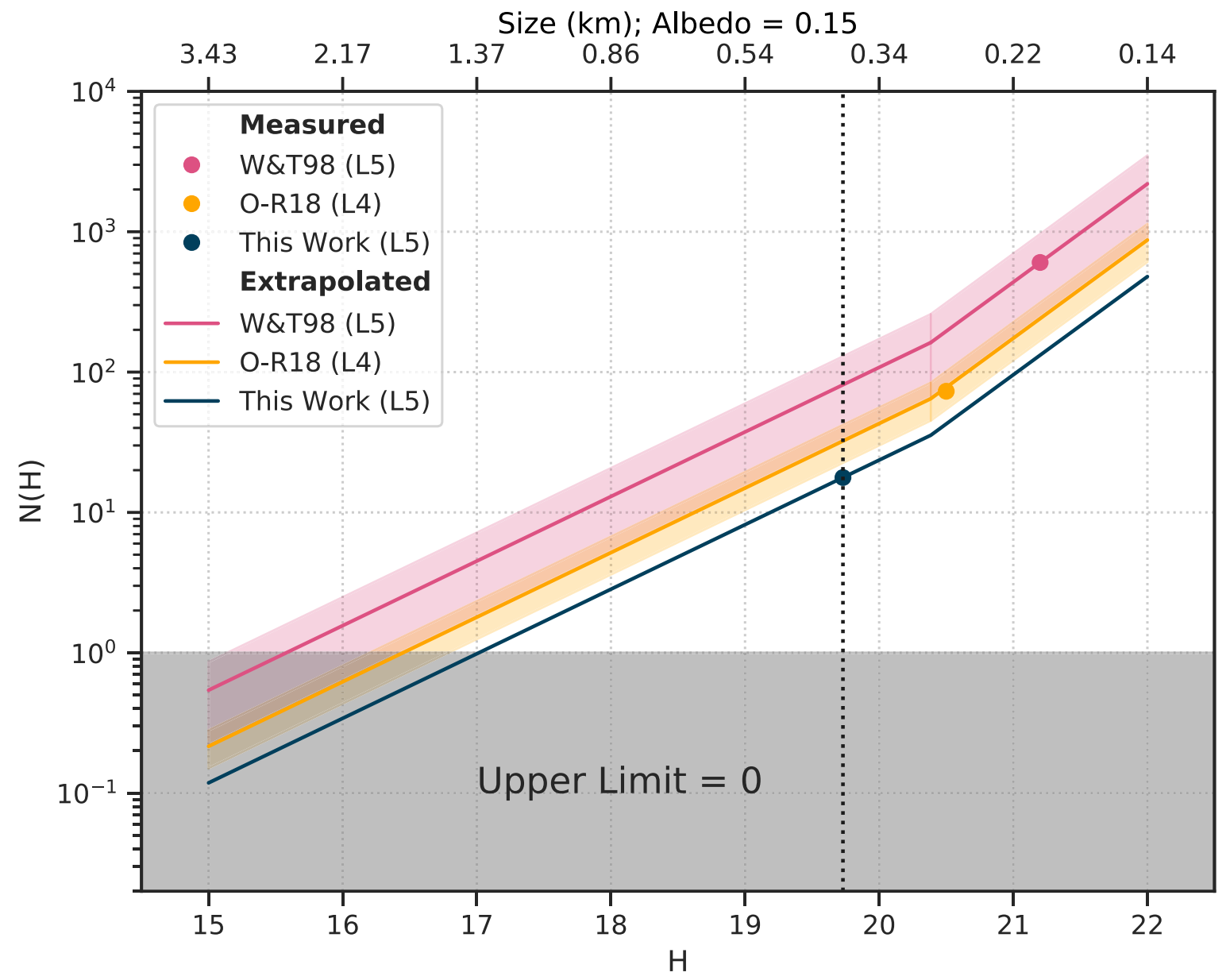

Figure 12. Extrapolated upper limits (lines) based on the measured upper limit (circular points) for each survey. The results for this work are in blue, Cambioni et al. (2018) in yellow, and the upper limit calculated by Cambioni et al. (2018) based on the results in Whiteley \& Tholen (1998) are in pink. The dotted line represents the point used for extrapolation for the results in this work, $H=19.7$. The grey region depicts where $N(H)<1$, meaning the upper limit is essentially 0 ETs. The slope of the power law we use is $\alpha=0.46$ for $H<20.39$ and $\alpha=0.7$ for $H>20.39$, which gave the most conservative upper limits in Fig. 11 . Under these assumptions, the results from this work give the most stringent upper limit on the ET population to date (18 ETs with $H=19.7$ as compared to 32 and 81). For steeper power laws, the upper limits for this work are essentially consistent with O-R18.

However, in comparing to this single magnitude, we are comparing to our most poorly constrained upper limit. Therefore, we follow a process similar to that used in Cambioni et al. (2018) in which we extrapolate these other upper limits to a brighter $H$ for which our survey has better constrained upper limits by assuming a power-law distribution (see Section 5.3). This method requires assuming a slope parameter; we use $\alpha=0.46$ for $H<20.39$ and $\alpha=0.7$ for $H>20.39$ as these values give the most conservative upper limits in Fig. 11.

Fig. 12 shows the extrapolated upper limits using this model; for each survey, the circular point is the upper limit at the magnitude that we extrapolated from (coming from this work and Cambioni et al. 2018)). Again, our upper limits are clearly more stringent than the W\&T98 limits. Under these assumptions, our upper limits are also slightly more stringent than those from O-R18; if we used a power law with $\alpha=0.7$ only instead, our results are essentially consistent with the O-R18 results. At $H=19.7$, the point of extrapolation used for this work, we calculate an upper limit of 18, 32, and 81 ETs for this work, O-R18, and W\&T98, respectively. In short, although our survey does not have as faint of a limiting magnitude as the previous surveys, we are able to place more stringent limits on the L5 population at brighter magnitudes than Whiteley \& Tholen
(1998) and limits that are consistent to slightly more stringent than the OSIRIS-REx L4 search (Cambioni et al. 2018).

\subsection{Missing primordial ETs?}

After this work, the existence/characterization of primordial ETs is still an open question. Even though it is almost certainly not primordial, $2010 \mathrm{TK}_{7}$ remains the only known ET. All of the current upper limits on the ET population, including from this work, suggest that there could still be tens to hundreds (depending on size) of ETs outside of the survey area of four independent searches. Deeper and broader coverage of both Lagrange clouds is necessary to say definitively whether or not other ETs exist.

However, a dearth of additional ETs would not be entirely unexpected, especially for very faint/small objects. Numerical simulations have suggested that primordial ETs with $H>20.5$ (assuming an albedo of 0.1) are likely to be destabilized due to the Yarkovsky effect within 4.5 Gyr (Zhou et al. 2019). These results combined with previous non-detections of ETs lead Zhou et al. (2019) to conclude that it is very unlikely that primordial ETs of absolute magnitude $H<=20.5$ (or their equivalent size limit) 
exist at the Lagrange points today, which could explain the current paucity of ETs. This work, as well as previous surveys, are sensitive to objects primarily above this limit; thus, deeper searches would allow us to test this theoretical limit.

It is important to note that it is also possible for asteroids to be temporarily trapped in an ET-like orbit, like $2010 \mathrm{TK}_{7}$. Morais \& Morbidelli (2002) found that such objects are likely quite rare though; through their simulations they calculated an upper limit of $0.65 \pm 0.12$ and $16.3 \pm 3.0$ captured Earth co-orbitals for $H<18$ and $H<22$, respectively. Based on these results, it seems possible that $2010 \mathrm{TK}_{7}$ truly is the only Earth co-orbital. However, it is also possible that the Yarkovsky effect (not included in those simulations) could in fact improve the capture efficiency for transient co-orbital ETs (Malhotra 2019).

Ultimately, in the absence of stronger observational constraints, there is still little we can definitely say about primordial ETs. Broader and deeper searches are still needed in order to further constrain this population.

\section{ACKNOWLEDGEMENTS}

This material is based upon work supported by the National Aeronautics and Space Administration under Grant No. NNX17AF21G issued through the Solar System Observations Planetary Astronomy Program. This work is partially funded by Michigan Space Grant Consortium, National Aeronautics and Space Administration grant \#NNX15AJ20H. LM, JCB, and SJH are supported by the National Science Foundation Graduate Research Fellowship Grant No. DGE 1256260. RM acknowledges support from National Aeronautics and Space Administration (NExSS; grant NNX15AD94G).

Based in part on observations at Cerro Tololo InterAmerican Observatory, NSF's National Optical-Infrared Astronomy Research Laboratory, which is operated by the Association of Universities for Research in Astronomy (AURA) under a cooperative agreement with the National Science Foundation. These observations were taken under NOAO 2018A-0177, Alex Parker et al. This research has made use of data and/or services provided by the International Astronomical Union's Minor Planet Center.

We thank the referee for helpful and constructive comments, which improved this paper. We would like to thank Hsing Wen Lin and Tali Khain for their useful comments on this paper.

\section{REFERENCES}

Almeida A. J. C., Peixinho N., Correia A. C. M., 2009, A\&A, 508, 1021 Astropy Collaboration, 2013, A\&A, 558, A33

Astropy Collaboration, 2018, AJ, 156, 123

Berthier J., Vachier F., Thuillot W., Fernique P., Ochsenbein F., Genova F., Lainey V., Arlot J.-E., 2006, in Gabriel C., Arviset C., Ponz D., Enrique S., eds, ASP Conf. Ser. Vol. 351, Astronomical Data Analysis Software and Systems XV. Astron. Soc. Pac., San Francisco, p. 367

Bertin E., Arnouts S., 1996, A\&AS, 117, 393

Bottke W. F., Durda D. D., Nesvorný D., Jedicke R., Morbidelli A., Vokrouhlický D., Levison H., 2005, Icarus, 175, 111

Bottke W. F., Jedicke R., Morbidelli A., Petit J.-M., Gladman B., 2000, Science, 288, 2190

Cambioni S., et al., 2018, An Upper Limit on Earth's Trojan Asteroid Population from OSIRIS-REx, 49th Lunar and Planetary Science Conference. p. 1149

Connors M., Wiegert P., Veillet C., 2011, Nature, 475, 481

Ćuk M., Hamilton D. P., Holman M. J., 2012, MNRAS, 426, 3051

de la Fuente Marcos C., de la Fuente Marcos R., 2014, MNRAS, 439, 2970 de la Fuente Marcos C., de la Fuente Marcos R., 2017, MNRAS, 467, 1561

Dvorak R., Lhotka C., Zhou L., 2012, A\&A, 541, 127

Flaugher B. et al., 2015, AJ, 150, 150

Gallant J., Gladman B., Ćuk M., 2009, Icarus, 202, 371

Ginsburg A.et al., 2019, AJ, 157, 98

Harris A. W., D’ Abramo G., 2015, Icarus, 257, 302

Ito T., Malhotra R., 2010, A\&A, 519, A63

Ivezić Ž. et al., 2001, AJ, 122, 2749

Jedicke R., Metcalfe T. S., 1998, Icarus, 131, 245

JeongAhn Y., Malhotra R., 2010, BAAS, 42, 1053

JeongAhn Y., Malhotra R., 2015, Icarus, 262, 140

Jester S. et al., 2005, AJ, 130, 873

Lin H. W. et al., 2019, Icarus, 321, 426

Mainzer A. et al., 2012, ApJ, 752, 110

Malhotra R., 2019, Nat. Astron., 3, 193

Malhotra R., Jeongahn Y., 2011, EPSC-DPS Joint Meeting 2011, Copernicus, Göttingen, Germany, p. 1215

Marzari F., Scholl H., 2013, Celest. Mech. Dyn. Astron., 117, 91

Morais M. H. M., Morbidelli A., 2002, Icarus, 160, 1

Morbidelli A., Levison H. F., Tsiganis K., Gomes R., 2005, Nature, 435, 462

Morota T., Furumoto M., 2003, Earth Planet. Sci. Lett., 206, 315

Pedregosa F. et al., 2011, Journal of Machine Learning Research, 12, 2825

Rein H., Liu S.-F., 2011, A\&A, 537, A128

Tabachnik S. A., Evans N. W., 2003, MNRAS, 319, 63

Tsirvoulis G., Morbidelli A., Delbo M., Tsiganis K., 2018, Icarus, 304, 14

Valdes F., Gruendl R., DES Project, 2014, in Manset N., Forshay P., eds, ASP Conf. Ser. Vol. 485, Astronomical Data Analysis Software and Systems XXIII. Astron. Soc. Pac., San Francisco, p. 379

Whiteley R. J., Tholen D. J., 1998, Icarus, 136, 154

Yoshida F., Terai T., 2017, AJ, 154, 71

Yoshikawa M., Tsuda Y., Watanabe S., Tanaka S., Nakazawa S., Terui F., Saiki T., 2018, Mission Status of Hayabusa2, 49th Lunar and Planetary Science Conference. p. 1771

Zhou L., Xu Y.-B., Zhou L.-Y., Dvorak R., Li J., 2019, A\&A, 622, A97

\section{APPENDIX A: SURVEY POINTINGS}

Table A1. Survey Pointings. Columns: (1) Name of the field. There were eight distinct fields in this survey, observed eight times each; (2) RA of exposure centre (h); (3) Dec. of exposure centre (deg); (4) Start time of exposure (UTC). Every exposure was taken on 2018-06-16.

\begin{tabular}{lccc}
\hline Field & RA & Dec. & Time \\
\hline ET_0 & 148.2684 & 13.71537 & $22: 45: 35$ \\
ET_0 & 148.2686 & 13.71430 & $22: 46: 18$ \\
ET_1 & 146.8672 & 12.61325 & $22: 47: 02$ \\
ET_1 & 146.8663 & 12.61278 & $22: 47: 45$ \\
ET_2 & 148.6678 & 11.91204 & $22: 48: 28$ \\
ET_2 & 148.6661 & 11.91206 & $22: 49: 11$ \\
ET_3 & 150.0658 & 13.21204 & $22: 49: 54$ \\
ET_3 & 150.0649 & 13.21155 & $22: 50: 36$ \\
ET_4 & 151.8663 & 12.61203 & $22: 51: 19$ \\
ET_4 & 151.8647 & 12.61239 & $22: 52: 03$ \\
ET_5 & 150.4654 & 11.31283 & $22: 52: 46$ \\
ET_5 & 150.4657 & 11.31343 & $22: 53: 29$ \\
ET_6 & 152.2671 & 10.71340 & $22: 54: 12$ \\
ET_6 & 152.2659 & 10.71455 & $22: 54: 56$ \\
ET_7 & 153.6655 & 12.01450 & $22: 55: 38$ \\
ET_7 & 153.6665 & 12.01432 & $22: 56: 20$ \\
ET_0 & 148.2658 & 13.71357 & $22: 57: 16$ \\
ET_0 & 148.2666 & 13.71279 & $22: 58: 00$ \\
& & &
\end{tabular}


Table A1 - continued

\begin{tabular}{|c|c|c|c|}
\hline Field & RA & Dec. & Time \\
\hline ET_1 & 146.8659 & 12.61219 & $22: 58: 46$ \\
\hline ET_1 & 146.8652 & 12.61255 & $22: 59: 29$ \\
\hline ET_2 & 148.6659 & 11.91192 & 23:00:11 \\
\hline ET_2 & 148.6661 & 11.91251 & 23:00:53 \\
\hline ET_3 & 150.0671 & 13.21225 & 23:01:40 \\
\hline ET_3 & 150.0665 & 13.21313 & $23: 02: 23$ \\
\hline ET_4 & 151.8678 & 12.61350 & 23:03:06 \\
\hline ET_4 & 151.8686 & 12.61258 & $23: 03: 50$ \\
\hline ET_5 & 150.4693 & 11.31235 & 23:04:32 \\
\hline ET_5 & 150.4683 & 11.31208 & $23: 05: 15$ \\
\hline ET_6 & 152.2692 & 10.71229 & 23:05:57 \\
\hline ET_6 & 152.2691 & 10.71294 & 23:06:40 \\
\hline ET_7 & 153.6702 & 12.01269 & $23: 07: 22$ \\
\hline ET_7 & 153.6697 & 12.01314 & 23:08:04 \\
\hline ET_0 & 148.2691 & 13.71246 & 23:08:59 \\
\hline ET_0 & 148.2682 & 13.71292 & $23: 09: 41$ \\
\hline ET_1 & 146.8680 & 12.61210 & $23: 10: 24$ \\
\hline ET_1 & 146.8683 & 12.61332 & 23:11:07 \\
\hline ET_2 & 148.6696 & 11.91296 & $23: 11: 49$ \\
\hline ET_2 & 148.6698 & 11.91222 & $23: 12: 33$ \\
\hline ET_3 & 150.0690 & 13.21317 & $23: 13: 16$ \\
\hline ET_3 & 150.0684 & 13.21276 & $23: 13: 58$ \\
\hline ET_4 & 151.8679 & 12.61339 & $23: 14: 41$ \\
\hline ET_4 & 151.8673 & 12.61308 & $23: 15: 23$ \\
\hline ET_5 & 150.4674 & 11.31342 & $23: 16: 05$ \\
\hline ET_5 & 150.4680 & 11.31393 & $23: 16: 47$ \\
\hline ET_6 & 152.2689 & 10.71375 & $23: 17: 29$ \\
\hline ET_6 & 152.2697 & 10.71322 & $23: 18: 12$ \\
\hline ET_7 & 153.6695 & 12.01254 & $23: 18: 53$ \\
\hline ET_7 & 153.6707 & 12.01305 & $23: 19: 37$ \\
\hline ET_0 & 148.2691 & 13.71227 & $23: 20: 34$ \\
\hline ET_0 & 148.2684 & 13.71222 & $23: 21: 17$ \\
\hline ET_1 & 146.8683 & 12.61170 & $23: 21: 59$ \\
\hline ET_1 & 146.8674 & 12.61258 & $23: 22: 43$ \\
\hline ET_2 & 148.6676 & 11.91242 & $23: 23: 25$ \\
\hline ET_2 & 148.6669 & 11.91336 & $23: 24: 08$ \\
\hline ET_3 & 150.0660 & 13.21263 & $23: 24: 52$ \\
\hline ET_3 & 150.0669 & 13.21335 & $23: 25: 35$ \\
\hline ET_4 & 151.8677 & 12.61336 & $23: 26: 17$ \\
\hline ET_4 & 151.8668 & 12.61394 & $23: 27: 01$ \\
\hline ET_5 & 150.4666 & 11.31324 & $23: 27: 43$ \\
\hline ET_5 & 150.4675 & 11.31401 & $23: 28: 26$ \\
\hline ET_6 & 152.2673 & 10.71314 & $23: 29: 09$ \\
\hline ET_6 & 152.2673 & 10.71354 & $23: 29: 51$ \\
\hline ET_7 & 153.6683 & 12.01315 & $23: 30: 34$ \\
\hline ET_7 & 153.6688 & 12.01308 & $23: 31: 16$ \\
\hline
\end{tabular}

\section{APPENDIX B: CALCULATING UPPER LIMITS UNDER DIFFERENT ASSUMPTIONS OF THE ET POPULATION}

In Section 5.3, we calculated various upper limits on the ET population, which required making several assumption about the population, namely its physical extent and size distribution. Under different assumptions, the calculated upper limits could deviate from what was presented here. Therefore, we aim to provide enough information such that the reader could calculate upper limits based on our observations but under their own assumptions.

We calculated upper limits from our recovery rates using equation (7). This equation depends on our calculated recovery rates and our assumed survey coverage of the L5 cloud. We determined this coverage to be 24 percent based on our synthetic population (see Section 5.1). However, these upper limits may be calculated with a different coverage value but the same measured values of the recovery rates. These values appear in Fig. 9, but we include them explicitly here in Table B1.

We also assume that the ET $H$ size distribution follows a power law (equation 5) in order to calculate even more stringent upper limits on the ET population, particularly for bright/large objects. We performed the extrapolation using the non-cumulative powerlaw distribution normalized to the upper limit we calculated for our point of extrapolation

$N(H)=\frac{U\left(H_{\mathrm{e}}\right)}{10^{\alpha H_{\mathrm{e}}}} * 10^{\alpha H}$,

where $H$ is the absolute magnitude, $H_{\mathrm{e}}$ is the extrapolation point, $U\left(H_{\mathrm{e}}\right)$ is the upper limit calculated at that extrapolation point, and $\alpha$ is a slope parameter. In Section 5.3, we use $H_{\mathrm{e}}=19.7, U\left(H_{\mathrm{e}}\right)=18$, and $\alpha=0.46$ for $H<20.39$ and $\alpha=0.7$ for $H>20.39$. However, this method may still be used for different assumptions of slope values, extrapolation points, or other functional forms of the ET $H$ distribution given our calculated upper limit at the $H$ bin used for extrapolation; these values appear in Fig. 10, but we include them explicitly here in Table B1.

Table B1. Values needed to calculate ET upper limits. Columns: (1) $H$ bin; (2) Recovery rate of injected Earth Trojans (see Sections 5.2 and 5.3) such that $1=100$ per cent of objects were recovered. If this column contains a dashed line, there were no injected objects in that $H$ bin; (3) Calculated upper limit without extrapolation for the number of ETs in each $H$ bin (see Section 5.3). If this column contains a dashed line, there were no injected objects in that $H$ bin, so we could not calculate an upper limit.

\begin{tabular}{lcc}
\hline$H$ & $R(H)$ & $U(H)$ \\
\hline 15.11 & 0.40 & 31.54 \\
15.33 & - & - \\
15.55 & 1.00 & 12.62 \\
15.77 & - & - \\
15.99 & 0.33 & 37.85 \\
16.21 & - & - \\
16.43 & 1.00 & 12.62 \\
16.65 & 0.50 & 25.23 \\
16.87 & 0.67 & 18.92 \\
17.09 & 0.83 & 15.14 \\
17.31 & 0.89 & 14.19 \\
17.53 & 0.67 & 18.92 \\
17.75 & 0.80 & 15.77 \\
17.97 & 0.90 & 14.02 \\
18.19 & 0.94 & 13.36 \\
18.41 & 0.75 & 16.82 \\
18.63 & 0.91 & 13.92 \\
18.85 & 0.77 & 16.33 \\
19.07 & 0.84 & 14.94 \\
19.29 & 0.62 & 20.19 \\
19.51 & 0.76 & 16.68 \\
19.73 & 0.71 & 17.73 \\
19.95 & 0.53 & 24.01 \\
20.17 & 0.31 & 40.27 \\
20.39 & 0.13 & 97.15 \\
\hline & &
\end{tabular}

This paper has been typeset from a $\mathrm{T}_{\mathrm{E}} \mathrm{X} / \mathrm{L} \mathrm{A} \mathrm{E} \mathrm{X}$ file prepared by the author. 